\title{
Improved Power Transformer Condition Monitoring under Uncertainty through Soft Computing and Probabilistic Health Index
}

\author{
J. I. Aizpurua a,*, B. G. Stewart ${ }^{\mathrm{b}}$, S. D. J. McArthur ${ }^{\mathrm{b}}$, B. Lambert ${ }^{\mathrm{c}}$, J. G. Cross ${ }^{\mathrm{d}}$, V. M. Catterson ${ }^{\mathrm{e}}$ \\ ${ }^{a}$ Mondragon University, Electronics E Computer Science Department - Signal Theory E Communications, Arrasate, Spain \\ ${ }^{b}$ University of Strathclyde, Department of Electronic and Electrical Engineering - Institute for Energy E Environment, Glasgow, UK \\ ${ }^{c}$ Bruce Power, Kincardine, Canada \\ ${ }^{d}$ Kinectrics Inc., Toronto, Canada \\ ${ }^{e}$ BioSymetrics, Toronto, Canada
}

\begin{abstract}
Condition monitoring of power transformers is crucial for the reliable and cost-effective operation of the power grid. The health index (HI) formulation is a pragmatic approach to combine multiple information sources and generate a consistent health state indicator for asset management planning. Generally, existing transformer HI methods are based on expert knowledge or data-driven models of specific transformer subsystems. However, the effect of uncertainty is not considered when integrating expert knowledge and data-driven models for the system-level HI estimation. With the increased dynamic and non-deterministic engineering problems, the sources of uncertainty are increasing across power and energy applications, e.g. electric vehicles with new dynamic loads or nuclear power plants with de-energized periods, and transformer health assessment under uncertainty is becoming critical for accurate condition monitoring. In this context, this paper presents a novel soft computing driven probabilistic HI framework for transformer health monitoring. The approach encapsulates data analytics and expert knowledge along with different sources of uncertainty and infers a transformer HI value with confidence intervals for decision-making under uncertainty. Using real data from a nuclear power plant, the proposed framework is compared with traditional HI implementations and results confirm the validity of the approach for transformer health assessment.
\end{abstract}

Keywords: Condition monitoring, transformer health monitoring, uncertainty, health index

\section{Introduction}

The correct and reliable operation of power and energy systems strongly depends on the health and condition of power transformers [1,2]. In safety-critical industries, such as nuclear power plants (NPPs), the safe and reliable generation of electricity to support industrial, residential and commercial loads is crucial. Part of the generated electricity is used for internal operation purposes and therefore, the availability of electricity is a critical requirement in a nuclear power plant (NPP) [3].

Condition monitoring and maintenance planning of power transformers is of vital importance for asset managers because their failure can lead to lack of export capability or even to catastrophic failures, e.g. see health monitoring applications in [4-8]. However, transformer health state estimation is a difficult task because it is comprised of complex and interacting subsystems. Figure 1 shows a simplified transformer Fault Tree Analysis (FTA) model [9] with some of its possible failure modes.

The transformer FTA model in Figure 1 relates low-level transformer faults, i.e. basic events, with the systemlevel transformer failure event using failure logic, i.e. OR gates. For example, winding assembly failure is caused by winding failure, connector failure or insulation breakdown. The specification of basic events is performed through failure rates or their corresponding degradation specifications.

\footnotetext{
${ }^{*}$ Corresponding author

Email address: jiaizpurua@mondragon.edu (J. I. Aizpurua)
} 


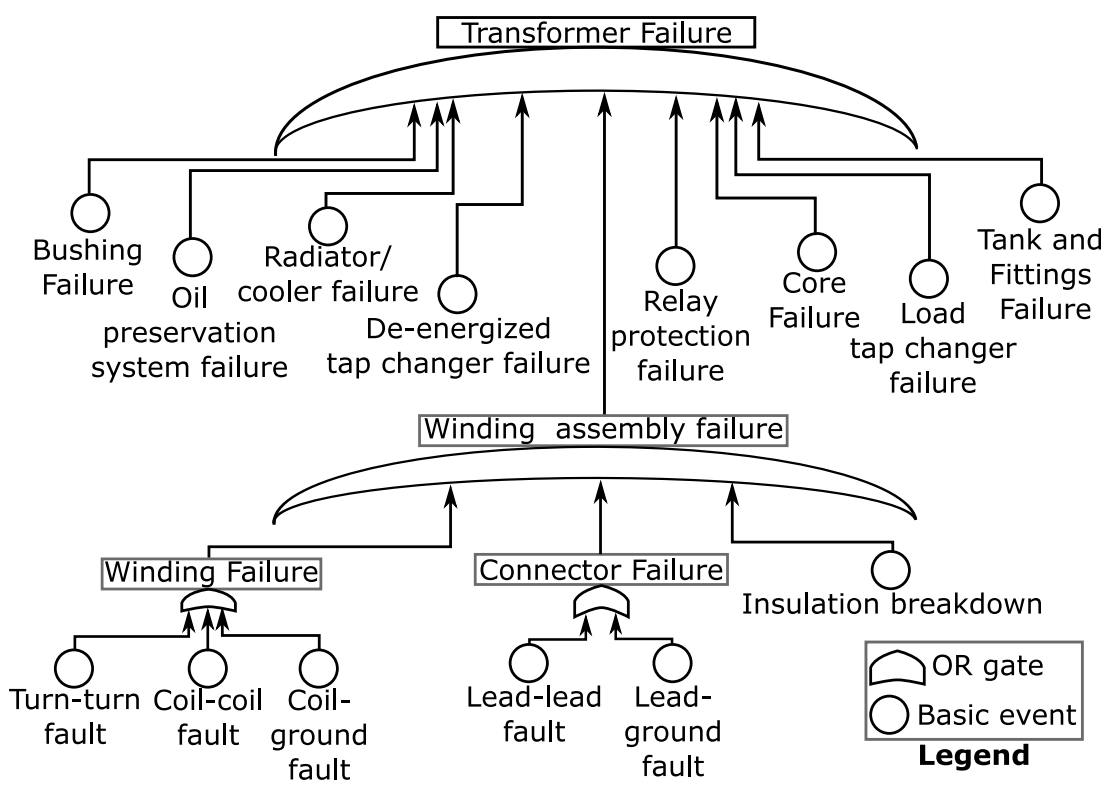

Figure 1: Transformer Fault Tree Analysis model [10].

The degradation mechanism of different transformer subsystems is intricate and surrounded by different sources of uncertainty. For instance, one of the key degradation factors of oil impregnated power transformers is the insulation breakdown. The degradation of the insulation is largely determined by the hottest-spot temperature, which is not directly measured, but commonly inferred from other measurements. In turn, the heat transfer process is distributed over different surfaces of the transformer which makes the estimation of the hottest-spot temperature more intricate.

In addition to the complex nature of transformers, there is an increased uncertainty in transformer operation and maintenance planning with the increased penetration of dynamic power and energy applications such as dynamic changes in electric vehicles [11] and smart grids [12] or de-energized periods in NPPs [13]. In this context, the nondeterministic and non-operational states directly affect the transformer nameplate rating and its useful life. In these cases, traditional methods used to calculate the transformer health state may not be accurate because they are based on analytic equations [14] and it is difficult to generalize with an analytical relation the properties that may affect the lifetime of the transformer and account for dynamic operational changes [15].

Accordingly, existing models for transformer health state estimation need to be revised to account for dynamic operation and maintenance changes along with the associated sources of uncertainty for a more accurate condition monitoring under conditions of uncertainty. With the increase of monitored parameters, data analytics and prognostics and health management (PHM) strategies have emerged as effective solutions to identify early indicators of anomalies, diagnose faults, and predict the remaining useful life (RUL) of different assets in the electrical power system [13, 1620]. However, it is necessary to integrate interacting PHM models from a systems engineering perspective for an accurate condition monitoring of complex systems such as power transformers.

In this context, the contribution of this paper is the proposal of a novel transformer health index framework which is able to take into account interactions between subsystems and different sources of uncertainty. The framework takes into account modelling uncertainties and variable specification uncertainties and propagates them to the final health index formulation and it facilitates:

- Maintenance planning. Analysis of the effect of different dissolved gases, oil quality, cooling system, load and temperature measurements on the transformer health state estimation.

- Decision making under uncertainty. Propagation and analysis of degradation modelling errors and variable specification errors on the transformer health state estimation.

- Risk monitoring. Analysis of the effect of different transformer subsystem degradation trends on the overall transformer health state estimation. 
The rationale for the design of the proposed health index framework emerges from reliability analysis of complex systems [9], i.e. FTA in Figure 1, complemented with machine learning and soft computing methods. To the best of the authors' knowledge this is the first attempt in combining these fields to improve transformer condition monitoring under uncertainty and obtain a thorough diagnostics model which includes confidence intervals for decision-making under uncertainty.

The remainder of this paper is organized as follows. Section 2 reviews related work. Section 3 presents the proposed soft computing driven transformer health index framework. Section 4 implements the approach in a real case study. Section 5 discusses the results and application, and finally, Section 6 draws conclusions and outlines future work.

\section{Related Work}

PHM strategies have been widely implemented to monitor the health of different power and energy components and systems such as bearings and turbofan engines [21] or wind turbine gearbox [22] (refer to [23] for a more exhaustive review). In the case of transformers, a common approach to address the transformer health state estimation is to use statistical lifetime models [24, 25] or health indices which combine information of different subsystems into a single transformer-level metric [7]. It is possible to include multivariate information with lifetime models (e.g., using covariates in Proportional Hazard Models [26]), but the focus of this paper is on the health index implementation based on the flexibility to integrate multiple information sources into a single health state indicator.

Jahromi et al. presented a health index approach which integrates different measurements including dissolved gas analysis (DGA), moisture, oil quality, furanic compound concentration, dissipation factor, leakage reactance, winding resistance, turn-ratio and core-to-ground resistance tests [27]. These parameters are compared with predefined static values to classify their condition, and then they are combined using expert knowledge. However, many of the decision rules to classify a monitored variable into a health index score are based on crisp boundary values.

Similarly, Tang et al. presented an evidential reasoning approach based on Analytical Hierarchy Process (AHP) and Dempster-Shafer (DS) theory. AHP integrates expert knowledge and DS theory combines subjective information of different subsystems and infers a single transformer health value [28].

In order to soften these decision bounds, Abu-Elanien et al. used fuzzy logic rules in the transformer health index specification [29]. The considered measurements include breakdown voltage (BDV) of the oil, total dissolved combustible gas values (TDCG), where TDCG $=\mathrm{H}_{2}+\mathrm{CH}_{4}+\mathrm{C}_{2} \mathrm{H}_{4}+\mathrm{C}_{2} \mathrm{H}_{2}+\mathrm{C}_{2} \mathrm{H}_{6}$, concentration of furans, dissipation factor (DF) of the oil, water content, acidity and 2-FAL. Similarly, Ashkezari et al. combined fuzzy logic with supervised classification methods [30] where firstly, the fuzzy logic module enables dealing with crisp decision bounds, and then a support vector machine (SVM) model classifies the transformer health into five predefined transformer health index groups.

Other authors have focused on specific transformer subsystems and they have used a data-driven approach for transformer condition monitoring. Ma et al. [17] presented a transformer monitoring framework including modules for denoising partial discharge (PD) signals and classifying faults through PD and DGA data. Li et al. [31] presented a probabilistic health index approach based on discrete Bayesian network (DBN) models. The significance of different measurements and maintenance data is determined based on principal component analysis (PCA) and expert knowledge. Then the DBN model is used to perform inferences and determine the transformer health state.

The selection of the most influential variables for transformer health index determination has also attracted the interest of many researchers. Tee et al. proposed a transformer ranking method through PCA and AHP [32]. The ranking criteria is the insulation condition and the monitored parameters include years in service, BDV, moisture, acidity, 2-FAL, DF, resistivity, interfacial tension (IFT) and colour. AHP integrates expert knowledge through pairwise comparisons of parameters, assigns weights and then PCA ranks parameters. Benhamed et al. presented a feature selection approach for health index specification [33]. The most influential variables are concentration of furan, water, acidity and BDV. Ibrahim et al. presented a selection criteria to choose the best parameters through an SVM classifier and optimization algorithms which are $\mathrm{CO}, \mathrm{C}_{2} \mathrm{H}_{2}, \mathrm{C}_{2} \mathrm{H}_{6}$ and furans [34].

With the introduction of new types of loads and applications such as electric vehicles, different sources of uncertainty may have a significant impact on the health index. Uncertainty quantification is very important for condition monitoring [35] and energy systems [36] and it is normally represented through probability density functions (PDF) of the likelihood of different estimates. 
In prior research and literature uncertainty-related methods have been focused on providing soft decision boundaries when combining transformer subsystem information via fuzzy logic $[29,30]$, inferring the health state from qualitative criteria via Dempster-Shafer theory [28], or defining a probabilistic framework through discrete probability values via DBN models [31].

However, there is no explicit consideration of uncertainty in the modelling process, e.g. lack of exact knowledge of the degradation model (known as systematic or aleatoric uncertainty) or uncertain variables in the degradation model, e.g. error-prone measurements. These sources of uncertainty directly affect the final transformer health state estimation.

Table 1 summarizes the reviewed works and their addressed key properties including:

(i) Integration of expert knowledge.

(ii) Determination of health indices through data analytics.

(iii) Combination of subsystem health indices into a transformer-level health index.

(iv) Uncertainty modelling.

Table 1: Reviewed health index works and addressed properties.

\begin{tabular}{|c|c|c|c|c|}
\hline Ref & Exp. Knowl. & Data-driven & Subsys. Comb. & Uncertainty \\
\hline$[17]$ & $\mathrm{x}$ & $\checkmark$ & $\checkmark$ & $\mathrm{x}$ \\
\hline$[27]$ & $\checkmark$ & $\mathrm{x}$ & $\checkmark$ & $\mathrm{x}$ \\
\hline$[28]$ & $\checkmark$ & $\mathrm{x}$ & $\checkmark$ & $\mathrm{x}$ \\
\hline$[29]$ & $\checkmark$ & $\mathrm{x}$ & $\checkmark$ & $\mathrm{x}$ \\
\hline$[30]$ & $\checkmark$ & $\checkmark$ & $\mathrm{x}$ & $\mathrm{x}$ \\
\hline$[31]$ & $\checkmark$ & $\checkmark$ & $\checkmark$ & $\mathrm{x}$ \\
\hline$[32]$ & $\checkmark$ & $\checkmark$ & $* \mathrm{R}$ & $\mathrm{x}$ \\
\hline$[33]$ & $\checkmark$ & $\checkmark$ & $* \mathrm{R}$ & $\mathrm{x}$ \\
\hline$[34]$ & $\checkmark$ & $\checkmark$ & $* \mathrm{R}$ & $\mathrm{x}$ \\
\hline Ours & $\checkmark$ & $\checkmark$ & $\checkmark$ & $\checkmark$ \\
\hline
\end{tabular}

${ }^{*}$ R: ranking of influential parameters.

There have been works focused on the quantification of the health state of the transformer. However, this is mainly based on expert knowledge [27, 28] or combination of expert knowledge with classification strategies [29, 30]. There has been research that presented intelligent applications for condition monitoring tasks [17], probabilistic health index models [31] and ranking the most influential variables for health index estimation [32-34].

The proposed condition monitoring models in Table 1 have been proven to be valid in their specific operation context. However, given the complex, dynamic and uncertainty surrounded nature of some of the emerging power and energy system applications such as smart grids and NPPs, it is necessary to complement knowledge-based, soft computing and probabilistic reasoning techniques with systems engineering concepts to evaluate the transformer health under uncertainty.

From a review of the current literature, there appears to have been no attempt to combine different subsystem data analytics with expert knowledge while taking into account the effect of uncertainties on the transformer health state determination. With the proposed modular bottom-up approach different failure modes and subsystems are analysed independently and then they are integrated at the transformer-level. The proposed modelling approach permits evaluating complex systems, subsystems, their interactions and associated uncertainty decisions. This is the direction of the proposed research work which distinguishes from other works in the field.

\section{A Soft Computing based Health Index framework for Transformer Condition Monitoring}

The proposed health index framework design process follows the methodology outlined in Figure 2a. The process starts from the data audit step by listing all the available datasets. Subsequently the diagnostics activity follows the 
process for the health state estimation of different transformer subsystems. The outcome of the diagnostics step is then converted into a normalized subsystem health index, and finally, different subsystem health indices are combined to create a transformer-level health index.

(a)

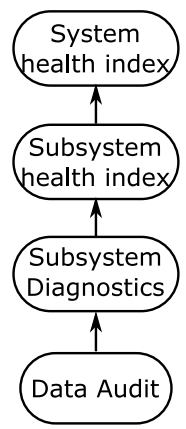

(b)

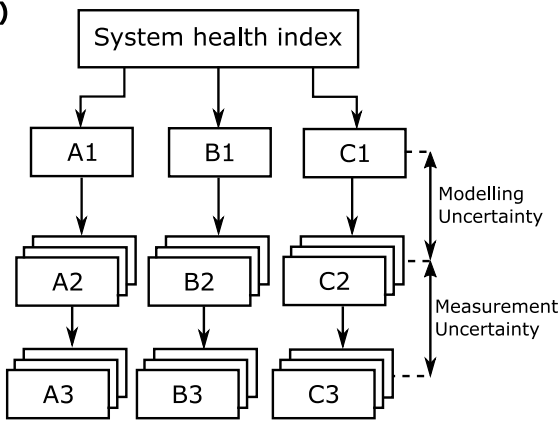

Figure 2: (a) Bottom-up health index modelling activities. (b) Top-down health index decomposition.

Figure $2 \mathrm{~b}$ shows the top-down health index decomposition with the corresponding datasets and models. Complex systems are comprised of different subsystems which are represented through their health index models (A1, B1, C1). The relation between systems and subsystems is determined from functional and non-functional dependencies. This can be defined using expert knowledge, e.g. using the logic failure modelled in the transformer FTA model in Figure 1.

Each subsystem may have more than one diagnostics model depending on the complexity of the subsystem (A2, B2, C2). The diagnostics activities estimate the health state of the transformer subsystem according to input datasets, sources of uncertainty and subsystem degradation models. The available datasets elicited from data audit step capture information related to the operation and degradation of different subsystems and they are used by different subsystem diagnostics models (A3, B3, C3).

The main sources of uncertainty are modelled at data source and diagnostics levels. Measurement uncertainties affect the available data sources with different errors such as measurement errors or quantization errors. Modelling uncertainties affect the designed diagnostics models with lack of exact knowledge or stochastic uncertainties.

Figure 3 shows the designed and implemented transformer health index framework taking the process shown in Figure 2 as a reference approach. Note that the available datasets and monitored subsystems are dependent on the specific condition monitoring strategy of each company.

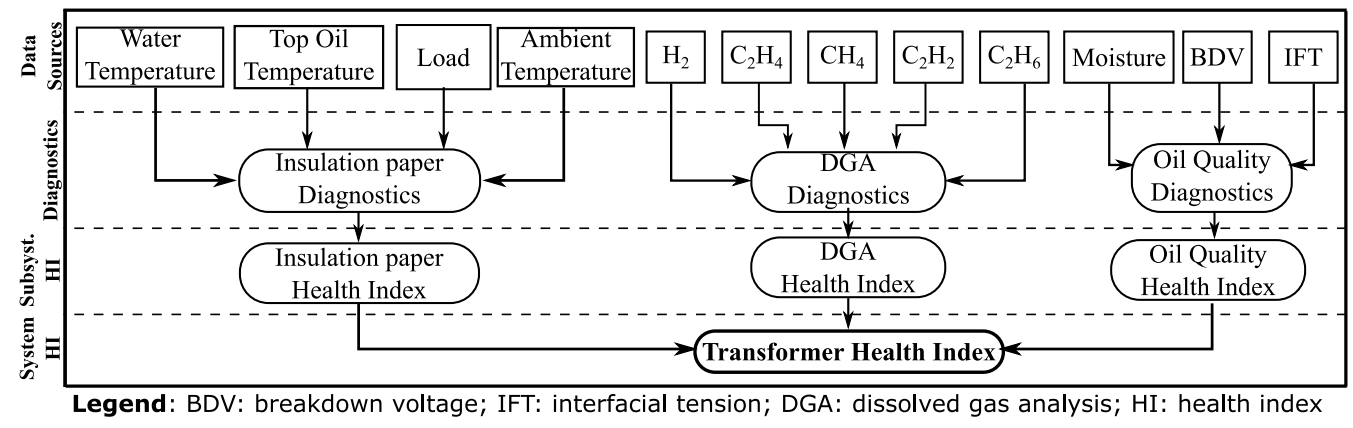

Figure 3: Soft computing driven transformer health index framework.

The available data sources are limited to dissolved gas analysis (DGA) samples $\left(\mathrm{H}_{2}, \mathrm{CH}_{4}, \mathrm{C}_{2} \mathrm{H}_{2}, \mathrm{C}_{2} \mathrm{H}_{4}, \mathrm{C}_{2} \mathrm{H}_{6}\right)$, load, ambient temperature, top-oil temperature, cooling water temperature, moisture in oil, BDV and IFT. As for the diagnostics models and subsystem health indices three modules have been implemented using these data sources: insulation paper health index, DGA based health index and oil quality based health index. The final step is to create a transformer health index which combines the subsystem health indicators.

The idea of combining subsystem data analytics and propagating different sources of uncertainties to obtain an uncertainty-aware transformer health index is novel. The proposed framework adapts the diagnostics outcomes of 
different subsystems and it combines them by propagating the different sources of uncertainty. Among the proposed subsystem data analytic models the DGA health index is a new approach and the insulation paper and oil quality health indices are based on previous diagnostics models [27, 37]. The DGA health index combines the diagnostics outcome of the presented uncertainty-aware DGA classification strategy with the absolute health index values inferred from IEEE standards (see Subsection 3.2).

\subsection{Insulation Paper Diagnostics $\mathcal{E}$ Health Index}

The life of the transformer insulation can be quantified by the degree of polymerization of the insulating paper. The rate of aging of the paper is primarily determined by the temperature. Namely, the cellulose chains of the paper are polymerised with heat, and over time, this leads to the paper becoming fragile, loss of insulation integrity and inability to withstand further stresses.

Aging is most rapid at the transformer hottest-spot and the paper will have here the lowest degree-of-polymerisation of the transformer. New paper has a degree-of-polymerization of approximately 1000-1200, while end of life is considered to be 200 [14].

\subsubsection{Transformer thermal and lifetime model}

IEEE C57.91 defines the insulation paper aging acceleration factor, $F_{A A}(t)$, as [14]:

$$
F_{A A}(t)=e^{\frac{15000}{383}-\frac{15000}{273+\Theta} H^{(t)}}
$$

where $\Theta_{H}(t)$ is the transformer winding's hottest-spot temperature in ${ }^{\circ} \mathrm{C}$, which can be calculated from other measurements [14]:

$$
\begin{aligned}
\Theta_{H}(t) & =\Theta_{T O}(t)+\Delta \Theta_{T O, H}(t) \\
& =\Theta_{A}(t)+\Delta \Theta_{A, T O}(t)+\Delta \Theta_{T O, H}(t)
\end{aligned}
$$

where $\Theta_{A}$ and $\Theta_{T O}$ are the ambient temperature and top-oil temperature and $\Delta \Theta_{A, T O}$ and $\Delta_{T O, H}$ are the top-oil and hottest-spot temperature rise over ambient and top-oil temperature respectively, which are calculated as follows in steady-state:

$$
\begin{aligned}
\Delta \Theta_{A, T O}(t) & =\Delta \Theta_{T O, R} \cdot\left[\left(\left(i(t) / i_{r}\right)^{2} \gamma+1\right) /(\gamma+1)\right]^{n} \\
\Delta \Theta_{T O, H}(t) & =\Delta \Theta_{H, R} \cdot\left(i(t) / i_{r}\right)^{2 m}
\end{aligned}
$$

where $\gamma$ is the ratio of load loss at rated load to loss at zero load, $i(t)$ is the transformer load, $i_{r}$ is the rated load, $\Delta \Theta_{T O, R}$ and $\Delta \Theta_{H, R}$ are the top-oil and hottest-spot temperature rise at rated load, and $m$ and $n$ are a transformer parameters determined through a lookup table depending on the cooling system of the transformer [14].

Finally Eq. (1) can be converted into a recurrence relation form so as to determine the remaining useful life (RUL) as follows:

$$
R U L(t)=R U L(t-1)-e^{\left(\frac{15000}{383}-\frac{15000}{273+\Theta_{H}(t)}\right)}
$$

Eq. (4) relates the insulation paper RUL with temperature and load measurements and guides the loading capability of the transformer by examining the effect of different load profiles on the transformer RUL. However, the application of Eq. (4) gives a single RUL value which does not consider the different uncertainties that affect the RUL estimation. Figure 4a shows a deterministic RUL estimate example with RUL=12 years at the prediction time instant of 3 seconds. In contrast, Figure $4 \mathrm{~b}$ shows a PDF estimate of the RUL value at the prediction time instant of 3 seconds with a maximum likelihood value of $1 \mathrm{e}-3$ for $\mathrm{RUL}=12$ years.

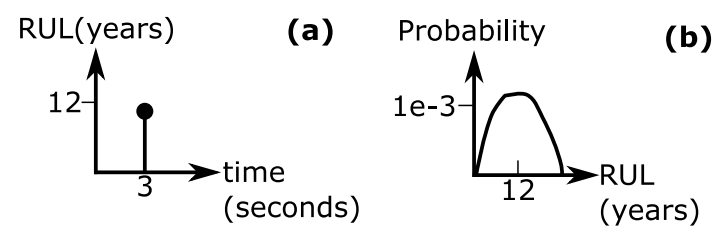

Figure 4: RUL prediction possibilities. (a) Deterministic estimate with RUL=12 years at $\mathrm{t}=3$ seconds; (b) RUL PDF at $\mathrm{t}=3$ seconds with RUL=12 years with a probability of $1 \mathrm{e}-3$. 
For a number of utilities it is common not to have hottest-spot temperature measurements because the measurement kit is not cost-effective. Thus the hottest-spot temperature estimation in Eq. (2) is inferred from indirect measurements.

Assuming that the top-oil temperature is measured, then the hottest-spot temperature calculated from top-oil temperature measurements may include measurement errors of top-oil temperature and load. Additionally, the initial health state and the paper consumption process [cf. Eq. (4)] may not be accurate due to the lack of knowledge and the stochastic behaviour of the paper degradation process. If these uncertainty-surrounded values are not considered the hottest-spot temperature estimation may lead to erroneous results.

Therefore, the effect of measurement errors needs to be explicitly considered. Accordingly Eq. (2), including measurement errors, is converted into:

$$
\Theta_{H}(t)=\left(\Theta_{T O}(t)+\varphi_{T O}\right)+\Delta \Theta_{H, R} \cdot\left[\left(i(t)+\varphi_{i}\right) / i_{r}\right]^{2 m}
$$

where $\varphi_{T O}$ denotes the top-oil measurement error and $\varphi_{i}$ designates the load measurement error.

Similarly, the paper degradation process in Eq. (1) is not a deterministic process and it also needs to integrate uncertainty information corresponding to the lack of exact knowledge of the degradation process:

$$
R U L(t)=R U L(t-1)+w_{R U L}-e_{t-1}^{\left(15000+w_{t}\right)\left(\frac{1}{383}-\frac{1}{273+\Theta_{H}(t)}\right)}
$$

where $w_{R U L_{t-1}}$ denotes the uncertainty of the lifetime estimation at $t-1, w_{t}$ denotes the degradation process uncertainty and $\Theta_{H}(t)$ is defined in Eq. (5). Initially $w_{R U L_{t-1}}$ will denote the initial lifetime estimation error, $w_{R U L_{0}}$, and then this error will be propagated in subsequent iterations through the recurrence relation form of Eq. (6).

The Particle Filtering framework outlined below effectively integrates these sources of uncertainty and tracks down the health state of the transformer over time according to the input data and the degradation model.

\subsubsection{Particle filtering $(P F)$}

$\mathrm{PF}$ is a Monte Carlo based Bayesian filtering method. PF enables the integration of multiple measurements in a single degradation model $f(\cdot)$ and filters the true state of the system, $x_{t}$, taking into account multiple sources of uncertainty [38]. A two-step method is implemented when PF is used for PHM [39, 40]. Firstly the system state estimation is performed:

$$
\begin{aligned}
x_{k} & =f\left(x_{k-1}, w_{k-1}\right) \\
z_{k} & =h\left(x_{k}, \varphi_{k}\right)
\end{aligned}
$$

where $f(\cdot)$ is the state degradation function defined in Eq. (6), $w_{k}$ is a state noise vector $w_{k}=\left\langle w_{t}, w_{R U L_{t}}\right\rangle, h(\cdot)$ is the measurement function defined in Eq. (5) and $\varphi_{k}$ is a measurement noise vector $\varphi_{k}=\left\langle\varphi_{T O}, \varphi_{i}\right\rangle$. The state-estimation $x_{k}$ given measurements $z_{k}$ up to the time instant $k$ is defined in terms of probability density function (PDF) $p\left(x_{k} \mid z_{0: k}\right)$.

The initial state $p\left(x_{0}\right)$ is assumed to be known. There are different methods to estimate the transformer initial state such as the experimental analysis of the degree of polymerization of the insulation paper, or if the paper is new, the initial health state may be assumed to be 180000 hours in the conditions stated in IEEE C57.91 [14]. Then the prior $P D F$ of the state $x_{k}$ from the distribution $p\left(x_{k-1} \mid z_{0: k-1}\right)$ is determined by:

$$
\begin{aligned}
p\left(x_{k} \mid z_{0: k-1}\right) & =\int p\left(x_{k} \mid x_{k-1}, z_{0: k-1}\right) p\left(x_{k-1} \mid z_{0: k-1}\right) d x_{k-1} \\
& =\int p\left(x_{k} \mid x_{k-1}\right) p\left(x_{k-1} \mid z_{0: k-1}\right) d x_{k-1}
\end{aligned}
$$

where the state-transition distribution function $p\left(x_{k} \mid x_{k-1}\right)$ is defined by the recurrence relation form in Eq. (7). In order to update the prior PDF, a new measurement is collected at time $k ; z_{k}$, and the posterior PDF is obtained using the Bayes rule:

$$
p\left(x_{k} \mid z_{0: k}\right)=\frac{p\left(x_{k} \mid z_{0: k-1}\right) p\left(z_{k} \mid x_{k}\right)}{p\left(z_{k} \mid z_{0: k-1}\right)}
$$

The analytic solution of Eq. (9) is complex. Thus, the PF was proposed based on iterative application of prediction, update and resampling steps at each time instant $k$ [38].

Prediction: assuming at time $k-1, N_{p}$ random samples (particles) of the system state are available, $\left\{x_{k-1}^{i}\right\}_{i=1}^{N_{p}}$, as a realization of the posterior distribution $p\left(x_{k-1} \mid z_{0: k-1}\right)$, the prediction at $k$ is performed by sampling the probability distribution of the system noise $w_{k-1}$ and simulating the system dynamics according to (7) to generate new samples $x_{k}^{i}$ which are realizations of the predicted distribution $p\left(x_{k} \mid z_{0: k-1}\right)$. 
$k$ :

Update: each sampled particle is assigned a weight based on the likelihoods of observations $z_{k}$ collected at time

$$
w_{k}^{i}=\frac{p\left(z_{k} \mid x_{k}^{i}\right)}{\sum_{j=1}^{N_{p}} p\left(z_{k} \mid x_{k}^{j}\right)}
$$

An approximation of the posterior PDF $p\left(x_{k} \mid z_{0: k}\right)$ is then obtained from the weighted samples $\left\{x_{k}^{i}, w_{k}^{i}\right\}_{i=1}^{N_{p}}$.

Resampling: as the PF evolves in time, weight degeneracy phenomena occurs where all but one particle have negligible weights [38]. To avoid this problem a degeneracy condition is defined based on the effective size: $\hat{N}_{\text {eff }}=$ $1 / \sum_{i=1}^{N_{p}} w_{k}^{i}$. If $\hat{N}_{e f f}$ falls below a threshold $N_{T}\left(N_{T}=N_{p} / 2\right.$ in this work), a systematic resampling step is applied [38].

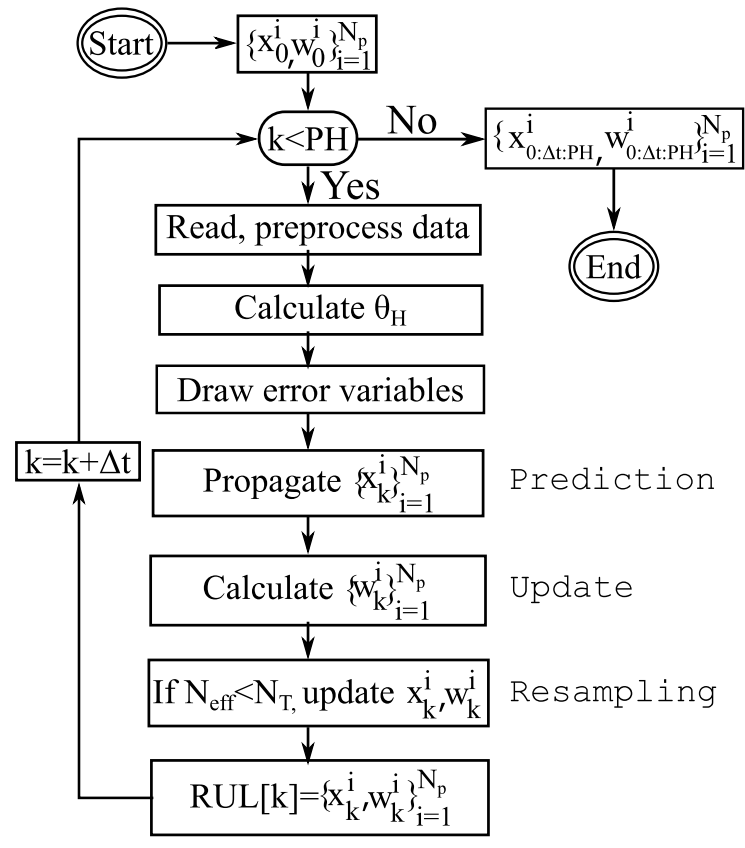

Legend: PH: prediction horizon, RUL: remaining useful life,

$\mathrm{x}_{\mathrm{k}}^{\mathrm{i}}$ : system state at time instant $\mathrm{k}$, trial $\mathrm{i}$;

$\mathrm{w}_{\mathrm{k}}^{\mathrm{i}}$ : particle weights at time instant $k$ trial $i$;

$\mathrm{N}_{\mathrm{p}}$ : number of particles; $\mathrm{N}_{\mathrm{T}}$ : threshold of number of particles;

$\mathrm{N}_{\text {eff: }}$ : effective size; $\theta_{\mathrm{H}}$ : hotspot temperature

Figure 5: Particle Filtering framework for paper lifetime estimation.

Figure 5 defines the implemented PF framework. $\Theta_{H}$ is calculated from Eq. (5). All the error variables are drawn from a Normal distribution: $r_{T O}=N\left(\Theta_{T O}, \varphi_{T O}\right), r_{i}=N\left(i, \varphi_{i}\right), r_{R U L_{k-1}}=N\left(R U L_{k-1}, w_{R U L_{k-1}}\right), r_{k}=N\left(0, w_{k}\right)$. The prediction step takes as input the estimated $\Theta_{H}(k)$ and $R U L(k-1)$ and calculates $\left\{x_{k}^{i}\right\}_{i=1}^{N_{p}}$. The process is repeated every timestep $\Delta t$ until reaching the prediction horizon $P H$.

The outcome of the PF approach is a PDF of the remaining paper lifetime estimated from the pair $\left\{x_{k}^{i}, w_{k}^{i}\right\}_{i=1}^{N_{p}}$, $\mathrm{k}=[0: \Delta t$ :Pred.Horizon $]$ (cf. Figure 4b). For consistency and post-processing purposes, the estimated RUL values are normalized with respect to the initial insulation paper health state $R U L_{0}$.

\subsection{DGA Diagnostics $\mathcal{E}$ Health Index}

Operational and fault events in the transformer generate gases which are dissolved in the oil that circulates through the transformer for cooling and insulation purposes [41]. DGA is an industry-standard method that focuses on the study of these gases. Traditional industry-accepted DGA methods classify the transformer health according to predefined gas ratios and intervals, e.g. Duval's triangle or Roger's ratios [41]. However, these methods can give conflicting outputs and it is not always possible to consistently determine the real diagnostics cause [4]. There have been 
proposed improved DGA classification methods based on machine learning (ML) algorithms which learn statistical patterns from data and then classify new DGA samples accordingly [4-6].

ML approaches for DGA overcome the limitations of traditional methods and they are helpful under certain conditions. However, there are some other variables that can improve the DGA health state estimation because normally the DGA classification result is not considered in isolation for transformer maintenance decision-making. In this context, a novel DGA health index is proposed which combines the following submodules:

(i) rate-of-change of fault gasses $(\triangle \mathrm{DGA})$;

(ii) absolute value of the gasses (DGA factor, DGAF);

(iii) probability of normal transformer degradation state inferred from a statistical ensemble-based classification model.

Figure 6 shows the DGA health index framework including the input key gasses, different processing modules and a combination strategy to obtain a single DGA health index value.

Legend: $\mathrm{H}_{2}$ : hydrogen; $\mathrm{CH}_{4}$ : methane; $\mathrm{C}_{2} \mathrm{H}_{4}$ : ethylene; $\mathrm{C}_{2} \mathrm{H}_{2}$ : acetylene; $\mathrm{C}_{2} \mathrm{H}_{6}$ : ethanol;

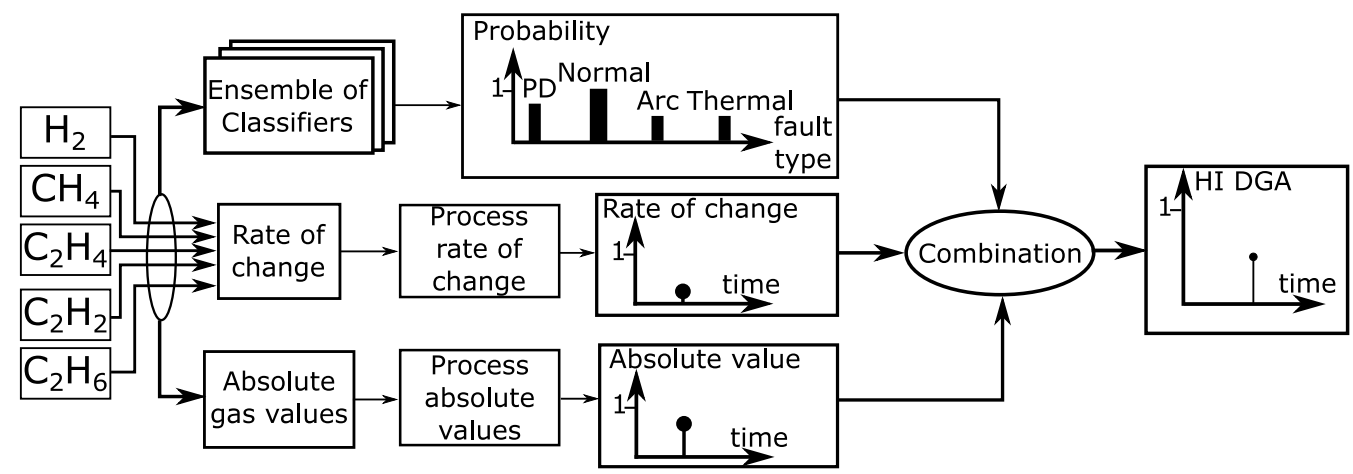

Figure 6: DGA health index framework.

The rate-of-change of gases enables the identification of abnormal situations over time. The absolute key gas values complement the transformer health state information with expert knowledge in the form of acceptable gas limits. The classification module determines the probability of normal transformer degradation state using statistical learning models and training DGA data. Finally, all these measurements can be combined into a single DGA health index function:

$$
H I_{D G A}=\mathcal{F}(D G A F, \triangle D G A, \operatorname{Pr}(\text { Normal }))
$$

where $\mathcal{F}($.$) denotes an equally weighted average function.$

\subsubsection{Rate-of-change of gases}

There are different criteria for the quantification of the rate-of-change of gases. The approach adopted in this paper is based on IEEE C57.104 [41] which defines conditions and actions based on TDCG values and the rate-of-change of gases as defined in Table 2. Gas levels are measured in parts per million (ppm) units.

Table 2: Rate-of-change quantification criteria [41].

\begin{tabular}{|c|c|c|}
\hline Cond. & TDCG levels (ppm) & TDCG rate (ppm/day) \\
\hline Cond. \#1 & $\leq 720$ & $\# 1.1: \leq 10 ; \# 1.2: 10$ to $30 ; \# 1.3: \geq 30$ \\
\hline Cond. \#2 & 721 to 1920 & $\# \mathbf{2 . 1}: \leq 10 ; \# 2.2: 10$ to $30 ; \# 2.3: \geq 30$ \\
\hline Cond. \#3 & 1921 to 4630 & $\# 3.1: \leq 10 ; \# 3.2: 10$ to $30 ; \# 3.3: \geq 30$ \\
\hline Cond. \#4 & $\geq 4630$ & $\# 4.1: \leq 10 ; \# 4.2: 10$ to $30 ; \# 4.3: \geq 30$ \\
\hline
\end{tabular}


The rate-of-change conditions in Table 2 are linearly weighted from 1 (\#1.1) down to 0 (\#4.3). This process quantifies the contribution of the rate-of-change to the DGA health index determination $(\triangle \mathrm{DGA})$.

This index can be seen as a criticality analysis of the developed gas event where the most critical state is denoted with health value 0 indicating a rapidly increasing gassing rate with a very high TDCG value.

\subsubsection{Absolute key gas values}

The equation to determine the DGA factor (DGAF) is based on absolute gas values and it is defined through a weighted sum [27]:

$$
D G A F=\frac{\sum_{j=1}^{5} S_{j} \times W_{j}}{\sum_{j=1}^{5} W_{j}}
$$

where $j$ denotes the different gasses, $S_{j}$ is the gas score and $W_{j}$ is the corresponding weight both defined in Table 3 . The weights are elicited from [27] and the scores from IEEE C57.104 [41].

Table 3: Scoring and weight factors for gas levels (all in ppm units).
\begin{tabular}{|c|c|c|c|c|c|c|c|}
\hline \multirow{2}{*}{ Gas } & & \multicolumn{7}{|c|}{ Score $\left(S_{j}\right)$} & \multirow{2}{*}{$W_{j}$} \\
\cline { 2 - 8 } & $\mathbf{1}$ & $\mathbf{2}$ & $\mathbf{3}$ & $\mathbf{4}$ & $\mathbf{5}$ & $\mathbf{6}$ & \\
\hline \multirow{2}{*}{$\mathrm{H}_{2}$} & $\leq 100$ & $\begin{array}{c}100- \\
200\end{array}$ & $\begin{array}{c}200- \\
300\end{array}$ & $\begin{array}{c}300- \\
500\end{array}$ & $\begin{array}{c}500- \\
700\end{array}$ & $\geq 700$ & 2 \\
\hline $\mathrm{CH}_{4}$ & $\leq 75$ & $\begin{array}{c}75- \\
125\end{array}$ & $\begin{array}{c}125- \\
200\end{array}$ & $\begin{array}{c}200- \\
400\end{array}$ & $\begin{array}{c}400- \\
600\end{array}$ & $\geq 600$ & 3 \\
\hline $\mathrm{C}_{2} \mathrm{H}_{6}$ & $\leq 65$ & $65-80$ & $\begin{array}{c}80- \\
100\end{array}$ & $\begin{array}{c}100- \\
120\end{array}$ & $\begin{array}{c}120- \\
150\end{array}$ & $\geq 150$ & 3 \\
\hline $\mathrm{C}_{2} \mathrm{H}_{4}$ & $\leq 50$ & $50-80$ & $\begin{array}{c}80- \\
100\end{array}$ & $\begin{array}{c}100- \\
150\end{array}$ & $\begin{array}{c}150- \\
200\end{array}$ & $\geq 200$ & 3 \\
\hline $\mathrm{C}_{2} \mathrm{H}_{2}$ & $\leq 3$ & $3-7$ & $7-35$ & $35-50$ & $50-80$ & $\geq 80$ & 5 \\
\hline
\end{tabular}

The score denotes the criticality for each gas level and the weight denotes the influence of each gas on the final DGAF value, i.e. $\mathrm{C}_{2} \mathrm{H}_{2}$ is the most critical gas value and $\mathrm{H}_{2}$ is the least critical gas value.

\subsubsection{Ensemble of Classifiers}

The transformer fault classification through DGA focuses on a supervised learning problem with five input gases $\left(\mathrm{H}_{2}, \mathrm{CH}_{4}, \mathrm{C}_{2} \mathrm{H}_{4}, \mathrm{C}_{2} \mathrm{H}_{2}, \mathrm{C}_{2} \mathrm{H}_{6}\right)$ and four possible health states (normal degradation, arcing fault, partial discharge fault and thermal fault).

Ensembles of classifiers have been used to avoid the bias and risk of errors of individual classifiers and improve the diagnostics accuracy and prediction stability, e.g. [42, 43]. Ensemble models require post-processing the outcome of individual classifiers so as to generate a consistent prediction, and to this end, again uncertainty quantification is very important.

For instance, assume that a model has been trained to classify certain faults. So long as the test data is comprised of faults which are similar to the trained model, it should return a prediction with high confidence. However, if the model is tested on an unseen fault class, the model should be able to quantify this with uncertainty levels, which can convey information about the confidence of the diagnosis of the model. This information is completely lost with black-box (BB) models, which only represent purely numerical connections, but they tend to have a high classification accuracy. Conversely, white-box (WB) models capture expert knowledge either as a causal model or through firstprinciple models. They generate the uncertainty associated with the decision-making process by quantifying the PDF of the likelihood of different diagnostics states. This function represents the strength of the diagnosis of the model, i.e. the wider the variance, the less the confidence in the diagnostics outcome and vice-versa.

In this context it is possible to combine the accuracy of $\mathrm{BB}$ models with the uncertainty information of $\mathrm{WB}$ models so as to improve the final classification accuracy. Figure 7 shows the implemented uncertainty-aware ensemble diagnostics framework with two BB source classifiers (artificial neural networks - ANN, support vector machines SVM) and a WB classifier, i.e. Gaussian Bayesian Networks (GBN). For each group of DGA data samples, ANN and SVM models generate deterministic probability estimates $\left(m_{A N N}, m_{S V M}\right)$, and the GBN model generates a probability 
density function for each fault type which is preprocessed to infer maximum likelihood information and uncertainty information $\left(m_{B N}, m_{U}\right)$.

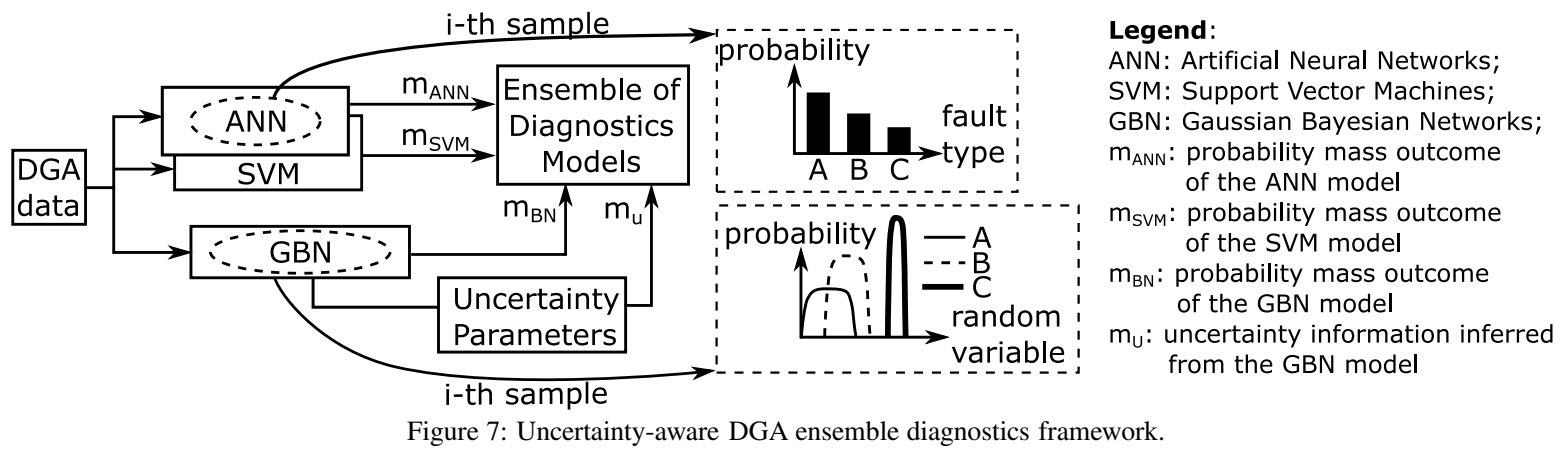

A combination of Dempster-Shafer theory and Bayesian methods is used to integrate all the source classifiers while taking into account the uncertainty information generated by WB probabilistic classifiers [44]. Assuming a set of faults $\mathcal{F}=\left\{f_{1}, \ldots, f_{i}, \ldots, f_{|F|}\right\}$ pieces of evidence are formulated as mass functions, $m: 2^{\mathcal{F}} \longmapsto \mathbb{R}$, satisfying: $m\left(f_{i}\right) \geq 0, m(\emptyset)=0$, and $\sum_{f_{i} \subseteq \mathcal{F}} m\left(f_{i}\right)=1$. The combined probability mass for the i-th fault, $f_{i}$, of two classifiers, denoted $c_{1}$ and $c_{2}$, taking into account the prior probability $\pi_{i}$ for each fault $(1 \leq i \leq|\mathcal{F}|)$ is calculated as:

$$
m_{c_{1} c_{2}}\left(f_{i}, \vec{\pi}\right)=\frac{m_{c_{1}}\left(f_{i}\right) \cdot m_{c_{2}}\left(f_{i}\right) \cdot \prod_{j=1}^{|\mathcal{F}| \backslash f_{i}} \pi_{j}}{\sum_{k=1}^{|\mathcal{F}|}\left(m_{c_{1}}\left(f_{k}\right) \cdot m_{c_{2}}\left(f_{k}\right) \prod_{l=1}^{|\mathcal{F}| \backslash f_{k}} \pi_{l}\right)}
$$

where $|\mathcal{F}|$ is the cardinality of the set of faults and $\vec{\pi}$ is the set of priors for each fault $\vec{\pi}=\left\{\pi_{1}, \pi_{2}, \ldots, \pi_{|F|}\right\}$.

The strength of the proposed reasoning framework is highlighted with conflicting data samples which are incorrectly classified by independent classifiers. In this situation, the prior information $\vec{\pi}$ is critical to weight the probabilities and decide which is the real cause of the fault.

The key assumption of this method is that the fusion method accepts a common prior for different mass values. That is, the uncertainty information inferred from a single classification method will be used to influence the combination of different classifiers. Therefore, the generation of representative uncertainty information will be critical. In the set of classifiers analyzed in this work, only the GBN model is able to generate uncertainty information from the classification output. Therefore, uncertainty parameters will be extracted from the density functions inferred by the GBN model so as to reason under uncertainty.

There are different metrics that can be used to extract uncertainty information from density functions such as standard deviation, kurtosis or entropy. Depending on the metric, the effect of the prior on the final accuracy is different. Best results were obtained with the standard deviation and weighted log-likelihood (wll):

$$
w l l=-\frac{1}{M} \sum_{i=1}^{M}\left(w_{i} \cdot p_{i}+\log \left(w_{i} \cdot p_{i}\right)\right)
$$

where $M$ denotes the total number of density samples, $p_{i}$ is the diagnosis probability of the fault $i$, and $w_{i}$ is the weight assigned to this probability.

So as to validate and generalize the results all the source models and the ensemble strategy have been examined $N=10^{3}$ times according to the Monte Carlo Cross Validation (MCCV) procedure, also known as random subsampling [45]. For each trial, firstly the dataset is randomly shuffled, then it is divided into $80 \%$ and $20 \%$ for training and testing sets, respectively. During the training stage, independent classifiers are trained by learning their corresponding hyperparameters. Subsequently, using the test data their corresponding independent diagnostics outputs are computed. Finally independent diagnostics outputs are combined through Eq. (13). After performing $N$ trials the mean and standard deviation accuracy statistics are quantified: 


$$
\hat{a}=\frac{1}{N} \sum_{i=1}^{N} a c c_{i} \quad s d_{\hat{a}}=\sqrt{\frac{1}{N} \sum_{i=1}^{N}\left(a c c_{i}-\hat{a}\right)^{2}}
$$

Table 4 reports the classification accuracy of the ensemble classification strategy along with another classical DGA method (Duval) as well as independent source classifiers (ANN, SVM, GBN) tested on the IEC TC 10 benchmarking dataset [46] through MCCV.

Table 4: Accuracy for the tested models on the IEC TC 10 dataset.

\begin{tabular}{|c|c|c|c|c|c|}
\hline \multirow{2}{*}{ Approach } & Overall & Thermal & $P D$ & $A r c$ & Normal \\
\cline { 2 - 6 } & $\hat{a} \pm s d_{\hat{a}}$ & $\hat{a} \pm s d_{\hat{a}}$ & $\hat{a} \pm s d_{\hat{a}}$ & $\hat{a} \pm s d_{\hat{a}}$ & $\hat{a} \pm s d_{\hat{a}}$ \\
\hline Duval & $67.8 \pm 7.2$ & $88.4 \pm 12.2$ & $\mathbf{1 0 0} \pm \mathbf{0}$ & $\mathbf{1 0 0} \pm \mathbf{0}$ & $0 \pm 0$ \\
\hline GBN & $82.1 \pm 6.3$ & $68.3 \pm 17.9$ & $97.3 \pm 9.4$ & $93.6 \pm 6.7$ & $72.9 \pm 14.9$ \\
\hline SVM & $86.6 \pm 6$ & $71.6 \pm 18.3$ & $93.1 \pm 17$ & $92.8 \pm 7.3$ & $87.6 \pm 11.2$ \\
\hline ANN & $89.4 \pm 5.3$ & $\mathbf{7 8 . 5} \pm \mathbf{1 6 . 5}$ & $91.6 \pm 18.5$ & $95.4 \pm 5.7$ & $88.7 \pm 10.7$ \\
\hline $\begin{array}{c}\text { Ensemble, } \\
\text { prior SD }\end{array}$ & $\mathbf{9 0 . 7} \pm \mathbf{5 . 2}$ & $\mathbf{7 8 . 5} \pm \mathbf{1 6 . 5}$ & $99 \pm 6$ & $96.3 \pm 5.4$ & $\mathbf{8 9 . 5} \pm \mathbf{1 0}$ \\
\hline $\begin{array}{c}\text { Ensemble, } \\
\text { prior WLL }\end{array}$ & $\mathbf{9 0 . 7} \pm \mathbf{5 . 2}$ & $\mathbf{7 8 . 5} \pm \mathbf{1 6 . 5}$ & $99 \pm 6$ & $96.3 \pm 5.4$ & $\mathbf{8 9 . 5} \pm \mathbf{1 0}$ \\
\hline
\end{tabular}

The best performing results in Table 4 are highlighted in bold. The overall accuracy of the proposed configurations are higher than other machine learning methods and traditional classification strategies which confirms the validity of the proposed classification approach. It may have been possible to use deep learning methods to further improve the accuracy of the classification model, e.g. [47], but in this case the capability to reason under uncertainty will be lost.

\subsection{Oil Quality Diagnostics $\mathcal{E}$ Health Index}

The focus of the oil health index is on weighted average health indices due to the lack of enough samples to create a representative statistical model (see Section 4). Namely, the oil health index is defined as follows [27]:

$$
\text { Oil Quality }=\frac{\sum_{k=1}^{5} S_{k} \times W_{k}}{\sum_{k=1}^{5} W_{k}}
$$

where $k$ denotes the different types of oil-related properties included in the oil health index definition such as IFT, $\mathrm{BDV}$, water content $\left(\mathrm{H}_{2} \mathrm{O}\right)$, colour and power factor (PFA).

Each of these parameters has a different weight, $W_{k}$, and score, $S_{k}$ as defined in Table 5 taken from the standard IEEE C57.106 [48]. The scores are classified into different intervals depending on the measured values and they denote the criticality for each oil factor. The weight denotes the influence of each factor on the final oil quality health index value. Finally, the health index value is normalized for post-processing purposes.

Table 5: Scoring and weight factors for oil levels.

\begin{tabular}{|c|c|c|c|c|c|}
\hline \multirow{2}{*}{ Gas } & \multicolumn{4}{|c|}{ Score $\left(S_{k}\right)$} & \multirow{2}{*}{$W_{k}$} \\
\cline { 2 - 5 } & $\mathbf{1}$ & $\mathbf{2}$ & $\mathbf{3}$ & $\mathbf{4}$ & \\
\hline IFT (dyne/cm) & $\geq 32$ & $25-32$ & $20-25$ & $<20$ & 2 \\
\hline $\mathrm{BDV}(\mathrm{kV})$ & $\geq 60$ & $50-60$ & $40-50$ & $<40$ & 3 \\
\hline $\mathrm{H}_{2} \mathrm{O}(\mathrm{ppm})$ & $\leq 15$ & $15-20$ & $20-25$ & $>25$ & 3 \\
\hline Colour & $\leq 1.5$ & $1.5-2$ & $2-2.5$ & $\geq 2.5$ & 2 \\
\hline Power Factor $(\%) 25^{\circ} \mathrm{C}$ & $\leq 0.1$ & $0.1-0.5$ & $0.5-1$ & $\geq 1$ & 3 \\
\hline
\end{tabular}




\subsection{Transformer Health Index}

The analytic equation for the transformer health index arises from the system failure logic condition. That is, the transformer failure will occur if any of its constituent subsystems fail with their corresponding occurrence probability.

The analytic equation for the transformer health index arises from the transformer failure logic condition. Namely, the failure of the transformer occurs when any of its constituent subsystem fails, i.e. failure of solid insulation, oil quality or DGA subsystems - see also the failure logic in the FTA model in Figure 1. The occurrence probabilities of different subsystems are weighted with their corresponding occurrence likelihood [27]:

$$
H I_{\text {Transfo }}=w_{1} \cdot H I_{\text {Paper }} \times w_{2} \cdot H I_{\mathrm{DGA}} \times w_{3} \cdot H I_{\text {Oil }}
$$

where $w_{1}, w_{2}$ and $w_{3}$ are respectively the weights corresponding to the solid insulation (5/13), DGA (5/13) and oil quality (3/13) subsystem failure conditions.

\section{Case Study}

There are different types of transformers in a NPP [1]. Figure 8 shows a example configuration of transformers in a NPP. This section examines the health state of a main output transformer which connects a generating station to the power grid.

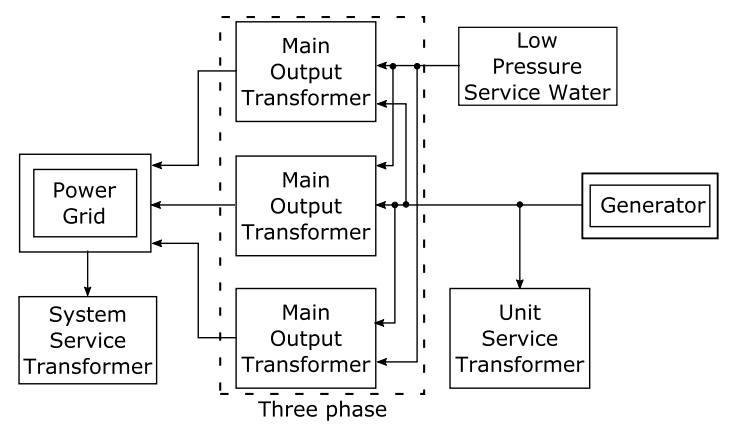

Figure 8: Example configuration of transformers in a NPP.

The proposed health index model captures different sources of uncertainty such as measurement uncertainties (possible load and temperature measurement errors), process uncertainties (errors associated with the degradation equation) and statistical uncertainties (see Section 3). These modelling mechanisms enable capturing the dynamic operation of transformers in NPPs with de-energized periods and maintenance outages.

The parameters of the main output transformer analysed in this section are reported in Table 6.

Table 6: Main output transformer parameters

\begin{tabular}{|c|c|c|c|}
\hline Param. & Value & Param. & Value \\
\hline Cooling $/ \mathrm{m}, \mathrm{n}$ & $\begin{array}{c}\text { Oil Directed Water } \\
\text { Forced } / 1,1\end{array}$ & Rating & $267 \mathrm{MVA}$ \\
\hline$\Delta_{H, R} / \Delta_{T O, R}$ & $30{ }^{\circ} \mathrm{C} / 24.3^{\circ} \mathrm{C}$ & $\mathrm{V}_{1} / \mathrm{V}_{2}$ & $17 \mathrm{kV} / 230 \sqrt{3} \mathrm{kV}$ \\
\hline$w_{\text {core,coil }} / w_{\text {tank }}$ & $95254 \mathrm{~kg} / 30617 \mathrm{~kg}$ & $i_{r} / \gamma$ & $15.1 \mathrm{kA} / 0.25$ \\
\hline
\end{tabular}

Note that the DGA health index has been validated and compared with other methods (cf. Table 4). The solid insulation health index is originated from IEEE standard equations [14] and the oil quality health index model is based on a weighted average method also originated from IEEE standards [48]. As a further validation and comparison strategy, for each of the analysed subsystems and for the overall transformer, the proposed health index approach and the traditional health index in [27] are implemented and compared. 


\subsection{Insulation paper health index}

According to the IEEE C57.91 standard the lifetime of newly installed insulation paper is approximately 180000 hours [14]. This is inferred from the degree of polymerisation of the transformer paper (see Subsection 3.1). In this section it is assumed that a Normal distribution models the uncertainty-related variables. As an example, in order to demonstrate the evaluation principles on the transformer, it is assumed that the initial health state is equal to new paper with 180000 hours with an uncertainty of 500 hours, i.e. $R U L_{0} \sim N(180000,500)$. Finally, the process noise (i.e., the uncertainty $w_{t}$ of the degradation model in Eq. (6)) is assumed to be $r_{k} \sim N(0,20)$. However, note that the PF framework is flexible and it enables the integration of non-Normal distributions too.

Figure 9 shows the monitored load and top-oil temperature data respectively. These datasets have been preprocessed through initial denoising and filtering steps. The power outages of the NPP match with zero load conditions and negative temperatures correspond with a long power outage in 2016 which resulted in negative top oil temperatures.

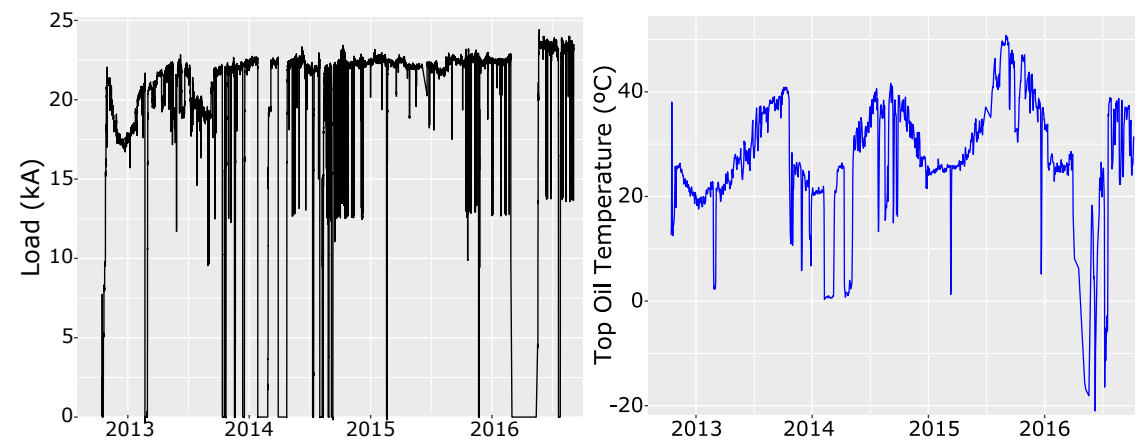

Figure 9: Monitored load and top-oil temperature data.

Using the Particle Filtering approach outlined in Figure 5, Figure 10 shows the remaining useful lifetime (RUL) estimation. The initial point in time is defined by the first available sample (11/2012). For the sensor errors, it is assumed that the temperature error is $\varphi_{T O}=5^{\circ} \mathrm{C}$ and the load error is $\varphi_{i}=1 \mathrm{kA}$. The operating time axis denotes the processed data (11/2012-09/2016), the RUL axis denotes the insulation paper degradation and the density axis denotes the PDF.

It is possible to observe in Figure 10 that instead of quantifying the health state around a single point value, the final health state is distributed into two nodes located at 169300 and 170200 hours. This is an effect of the random trajectory of the particles, i.e. independent simulations with parameters drawn from error distributions. This process results in a non-Normal PDF and therefore standard percentile values are not applicable. Instead, it is necessary to integrate the area under the curve to ensure the desired confidence interval (CI).

The inference of the confidence intervals facilitates the decision-making process because it enables engineers to adopt an uncertainty-informed decision with intuitive lower and upper limits on the estimated parameters. Note that the $95 \%$ confidence intervals have been used as an example, but the specification and inference of the confidence intervals is flexible and it can be defined by the engineers. Accordingly, taking all the results in Figure 10, the normalized mean health index values with $95 \%$ confidence intervals are shown in Figure 11. The normalization of the solid insulation paper diagnostics results is performed by dividing the RUL estimations in Figure 10 with the highest RUL prediction values.

The degradation is almost exponential as determined in Eq. (1), but this is also affected by the period during which the transformer was de-energized. For example, the transformer was shut down in mid-2016 for maintenance which resulted in zero load, decreased top-oil temperature and accordingly almost negligible RUL decrease.

Instead of using a paper health index Jahromi et al. account for the effect of load changes on the transformer health through a load factor (LF). The LF is based on the classification of load history into five groups [27]: $\mathrm{N}_{0}=$ number of $\mathrm{S}_{\mathrm{i}} / \mathrm{S}_{\mathrm{B}}$ that are lower than $0.6, \mathrm{~N}_{1}=$ number of $\mathrm{S}_{\mathrm{i}} / \mathrm{S}_{\mathrm{B}}$ that are between 0.6 and $1, \mathrm{~N}_{2}=$ number of $\mathrm{I}_{\mathrm{P}} / \mathrm{I}_{\mathrm{R}}$ that are between 1 and 1.3, $\mathrm{N}_{3}=$ number of $\mathrm{I}_{\mathrm{P}} / \mathrm{I}_{\mathrm{R}}$ that are between 1.3 and $1.5, \mathrm{~N}_{4}=$ number of $\mathrm{I}_{\mathrm{P}} / \mathrm{I}_{\mathrm{R}}$ that are greater than 1.5 , where $\mathrm{I}_{\mathrm{P}}$ is the monthly peak load and $I_{R}$ is the rated load of the transformer. Eq. (18) quantifies the LF and Figure 12 shows the application to the available dataset. 


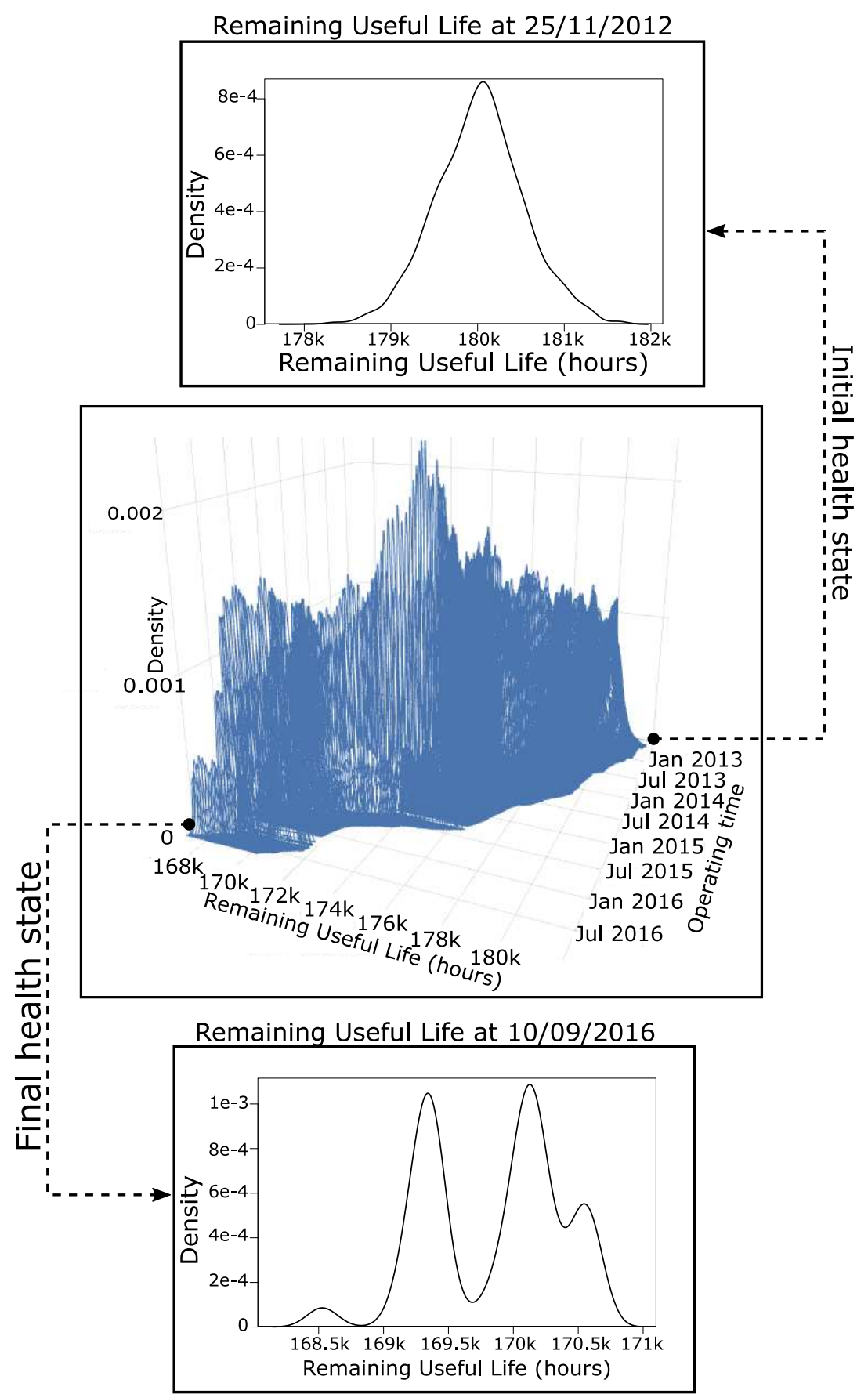

Figure 10: Transformer diagnostics results for the period 25/11/2012-10/09/2016.

$$
L F=\frac{\sum_{i=0}^{4}(4-i) \times N_{i}}{\sum_{i=0}^{4} N_{i}}
$$

It can be seen that the model in Eq. (18) is linear and it is directly affected by the monthly peak load. Therefore, the effect on the health index is more severe than the PF results in Figure 11.

The proposed solid insulation health index takes into account operational (load, oil temperature) and design param- 


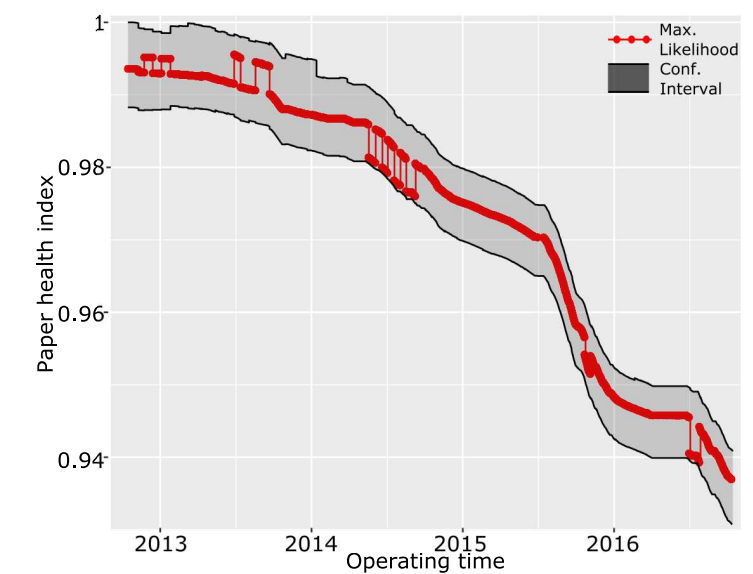

Figure 11: Solid insulation paper health index - 95\% CI of Figure 10.

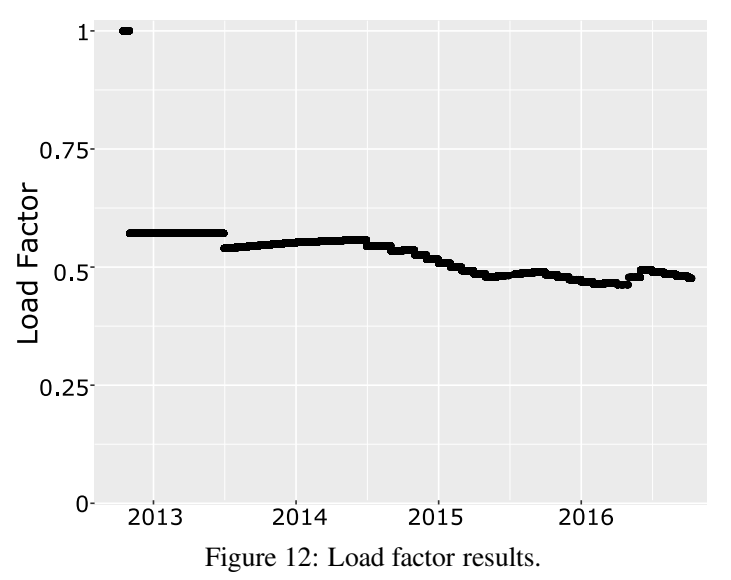

eters of the transformer. Additionally, the proposed Particle Filtering framework integrates the effect of measurement errors as well as stochastic parameters (e.g., the paper degradation process) on the final health index value. This results in a more informative solid insulation health index which also includes confidence intervals as shown in Figure 11.

It may have been possible to evaluate the solid insulation based on, e.g. fuzzy logic, but this would require introducing expert knowledge so as to deal with uncertainties. This paper adopts a data-driven Bayesian approach to model and propagate uncertainties from inspection data, removing the need for the capture of expert knowledge.

The confidence intervals model the uncertainty surrounding the maximum likelihood value. That is, if one does not consider the uncertainties outlined in Subsection 3.1 the final health estimation would be a deterministic estimate and the effect of measurement errors and unknown degradation processes will be lost (cf. Figure 4). In this context the Particle Filtering framework effectively integrates these parameters and generates confidence bounds. In this case the adopted uncertainty values result in the confidence bounds shown in Figure 11. Namely, the narrower the confidence bounds, the more confident the engineer will be in decision-making on a certain health index value, e.g. mid-2016. However, this is not always true and there are cases where the engineer should be aware of the possible boundary values so as to adopt an uncertainty-informed maintenance decision. This decision may be dependent on the criticality of the subsystem, i.e. in general, the more critical the subsystem, the more conservative will be the adopted maintenance decision.

\subsection{DGA heath index}

Figure 13 shows the available DGA dataset. The dataset covers the same time period as the load and top-oil temperature datasets (Figure 9), but the sampling rate is different. 


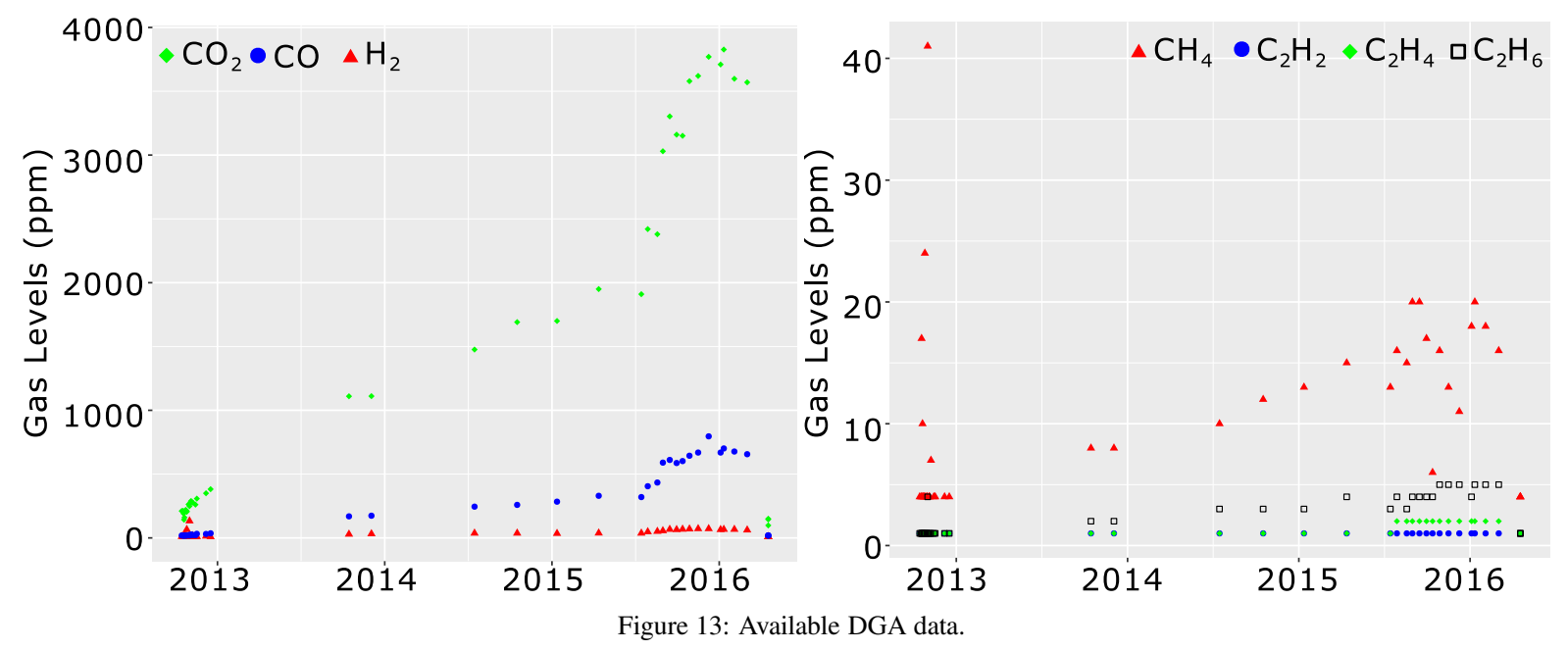

Figure 14 shows the effect of the rate-of-change of gases defined in Subsection 3.2.1 and Figure 15 shows the DGA factor results defined in Subsection 3.2.2.

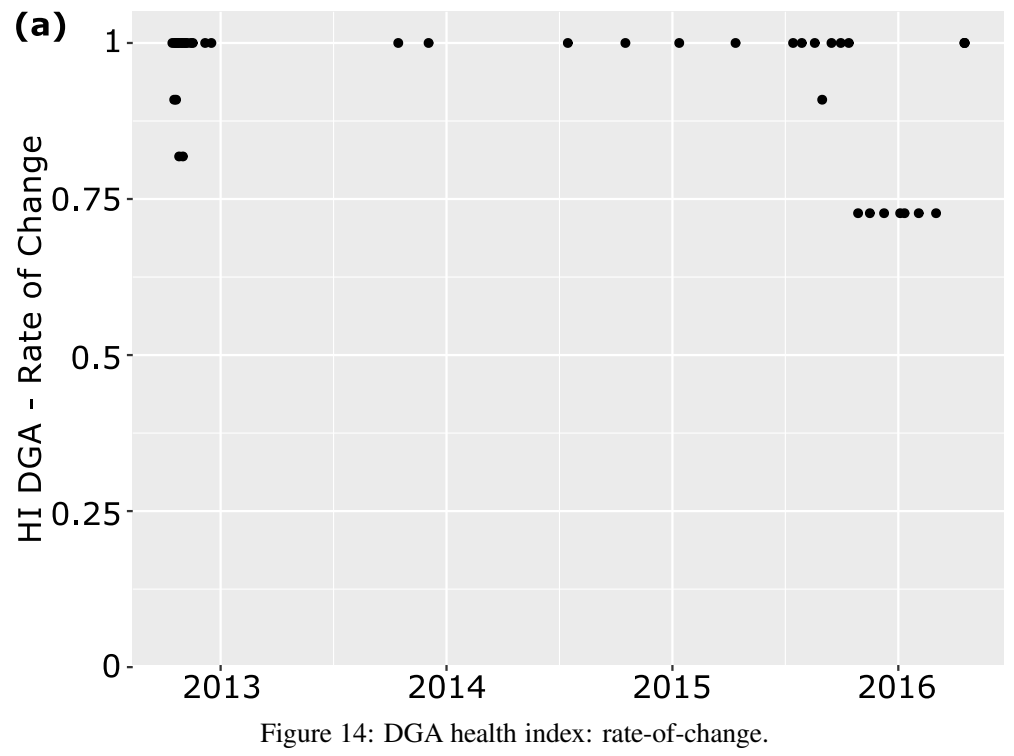

Most of the gas variations occur at the start and towards the end of the DGA dataset as seen in Figure 13 and confirmed in Figure 14. The gas values are within normal limits as indicated by the DGA factor in Figure 15. Only towards the end of the dataset the DGA factor decreases from the healthy state.

According to the ensemble classification strategy that is trained on an historical DGA dataset (Subsection 3.2.3), Figures 16 and 17 show the probabilistic diagnostics outcome corresponding to different health states, where D1 is a low energy discharge fault, D2 is a high energy discharge fault, T12 is a thermal fault with $\mathrm{T}<700^{\circ} \mathrm{C}$ and $\mathrm{T} 3$ is a thermal fault with $\mathrm{T}>700^{\circ} \mathrm{C}$.

It can be seen that up until 03/12/2013 most of the samples are classified as normally degrading transformers except some outliers located at 19/10/12, 21/10/12, 26/10/12,1/11/12,8/11/12 and 15/10/13. The diagnostics outcome from $03 / 12 / 2013$ onwards indicates a D1 fault which is later on diagnosed as T12 fault.

Figure 18 shows the DGA health index value by combining these measurements [cf. Eq. (11)]. The day distance label denotes the days between samples due to the irregular DGA sampling rate, i.e. the more days between samples, the larger the circular marker. 

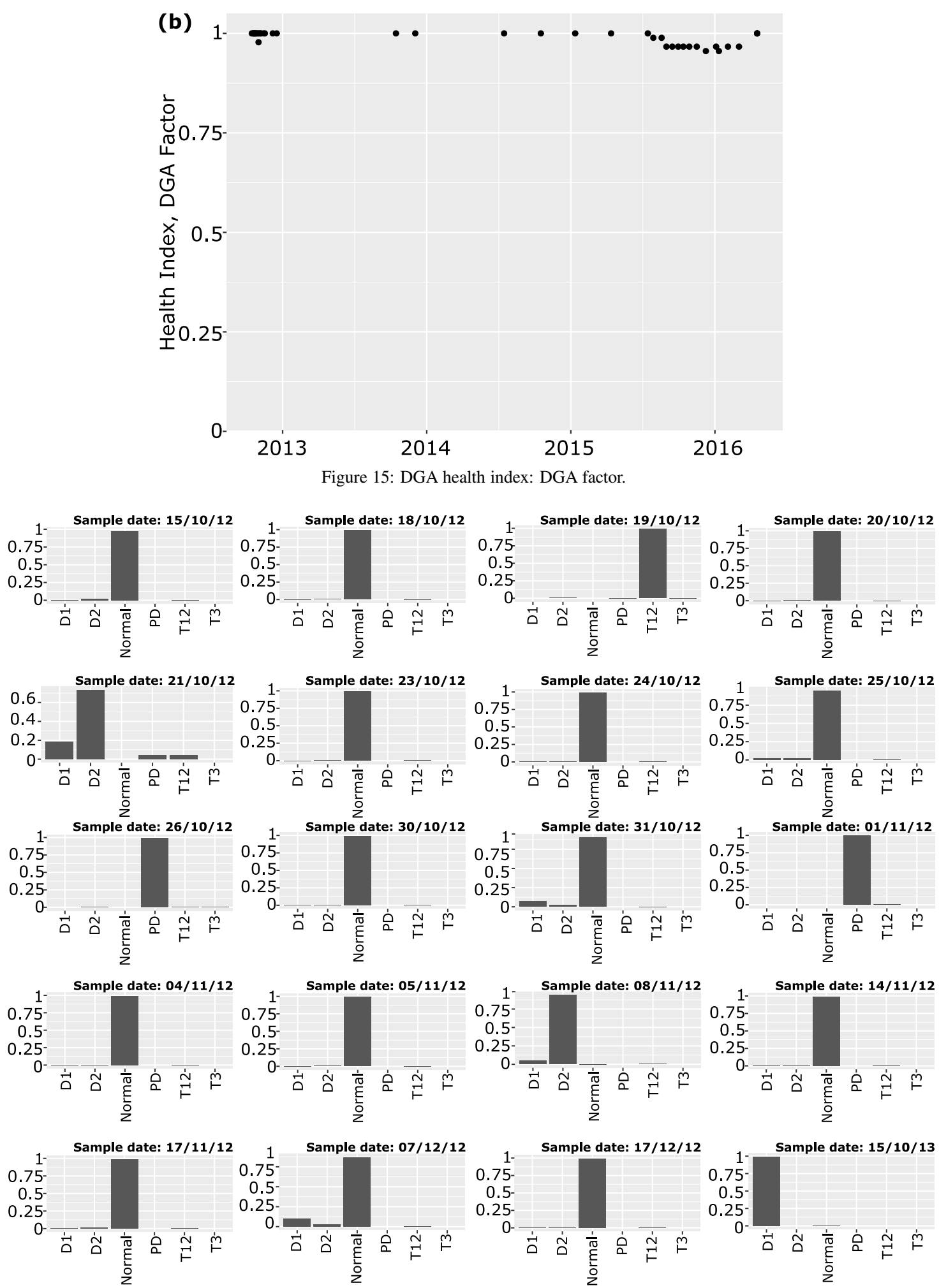

Figure 16: Health Index DGA: probabilistic diagnostics of the ensemble classification strategy. Sampling interval: 15/10/2012 — 15/10/13. 

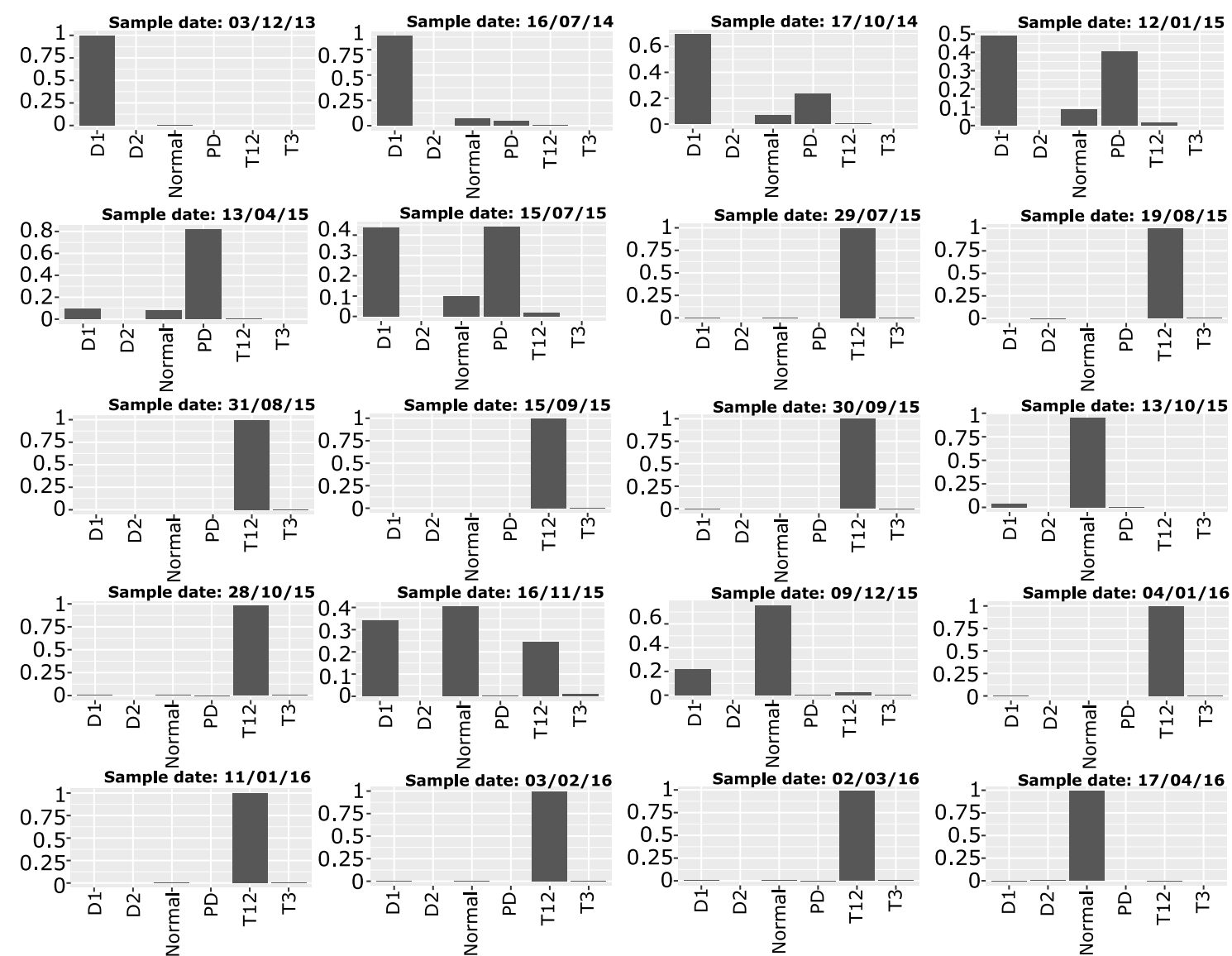

Figure 17: Health Index DGA: probabilistic diagnostics of the ensemble classification strategy. Sampling interval: 03/12/2013 — 17/04/16.

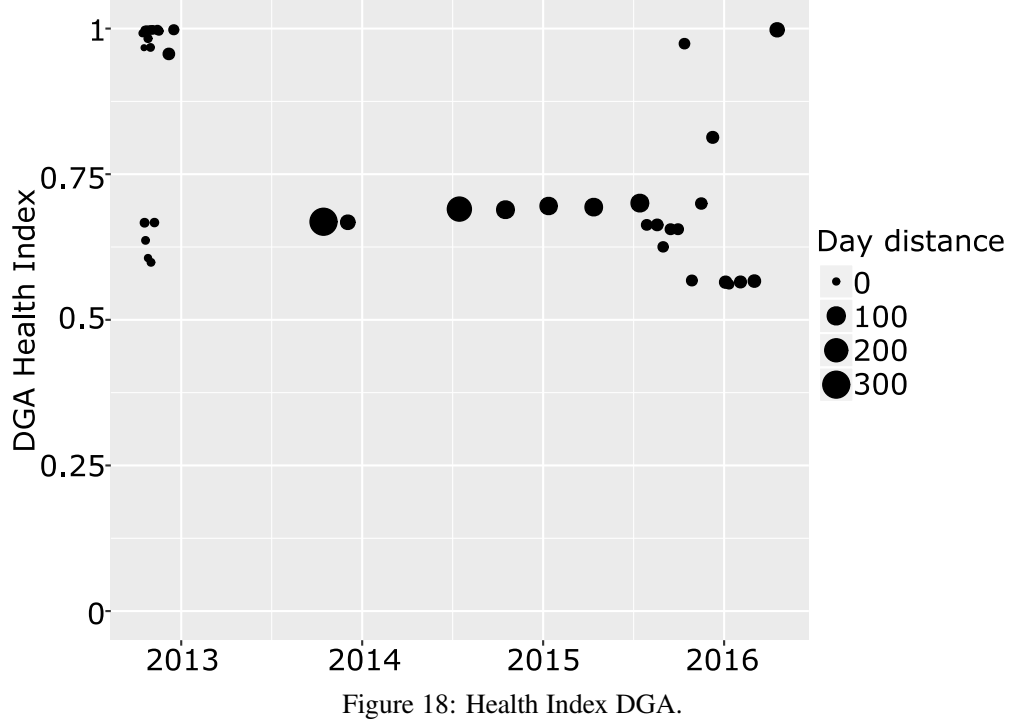

It is possible to identify four groups in Figure 18:

(i) Before 2013 there are two groups, one indicates a perfectly healthy transformer and another indicates a trans- 
former with a health index around 65\%. Looking at the rate-of-change and DGA diagnostics outcomes, one can see that some samples are not diagnosed as healthy and their rate-of-change does not indicate perfect health.

(ii) From 2013 until mid-2015 the sampling distance is very large. This may introduce more uncertainty in the diagnostics outcome due to the possible variations/errors in the DGA measurements. All these samples are around $70 \%$ health index. Both the rate-of-change and absolute gas values indicate a normal transformer. However, the DGA diagnostics outcome identifies a D1 fault for this period and this affects the final DGA health index.

(iii) From mid-2015 until April 2016 one can see that the DGA health index degrades over time. This is consistent across all three DGA health index modules: the rate-of-change drops below 75\%, the DGA factor decreases down to $90 \%$ and the DGA diagnostics outcome detects a T12 fault.

(iv) The last sample is diagnosed as a healthy sample by all the indicators.

The approach in [27] only makes use of the DGA factor which is shown in Figure 15b, with health index values greater than 0.95 for all the DGA factor results.

Note that the proposed DGA health index is a novel health index solution based on IEEE standards [41] and a probabilistic classifier which has been compared with different artificial intelligence and expert-based classification strategies (cf. Table 4).

The proposed DGA health index framework helps the engineer in the decision-making process by integrating additional variables that affect the DGA-based health state estimation. That is, in addition to the absolute gas values considered by traditional health index formulations, the rate-of-change of gases as well as historical DGA classification results based on statistical fusion strategies are also taken into account. All three indicators can be examined separately to confirm the root cause of a suspicious DGA health index value.

\subsection{Oil quality health index}

Figure 19 shows the available oil quality data samples.

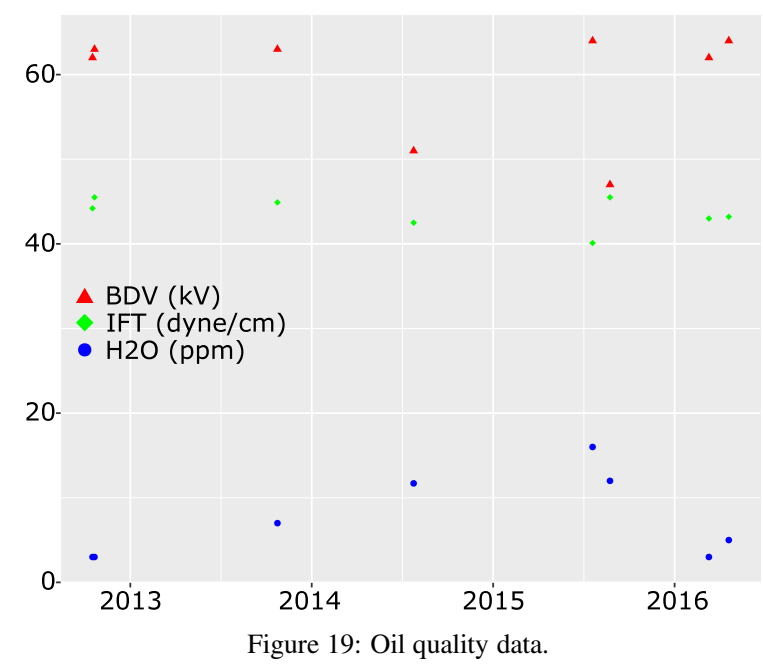

Figure 20 shows the oil health index results through the application of Eq. (16).

All the oil health index samples in Figure 20 show an excellent oil health status, except three samples located within 2014 and 2015 which are located at $85 \%, 90 \%$ and $92 \%$ health index values respectively. This module is the same for the traditional health index [27] and the proposed health index, and the results are identical. 

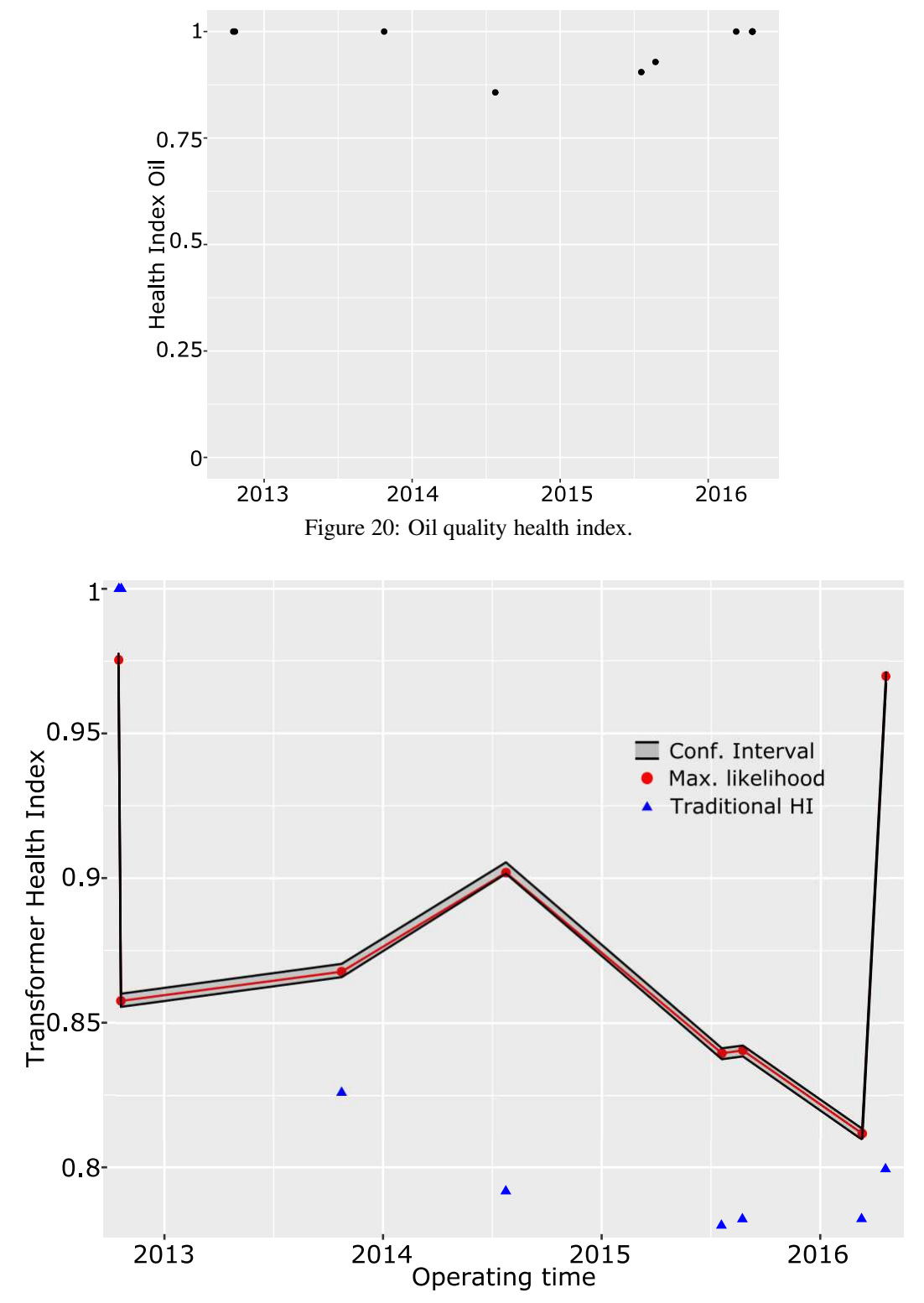

Figure 21: Traditional versus proposed transformer health index results.

\subsection{Transformer health index}

Figure 21 shows the maximum likelihood value and $95 \%$ confidence intervals of the proposed transformer health index and the results of the traditional health index in [27].

The results in Figure 21 are in agreement with previously defined DGA, oil and paper health indices. The DGA health index causes variations in these samples (Figure 18). The oil health index is mostly stable (Figure 20), and the solid insulation health index degrades at a slow rate (Figure 11).

The accuracy of the DGA diagnostics method is high (cf. Table 4), but there may be cases where the classifier can be wrong. The ensemble classification model shows the probability of being in all possible health states (see Figures 16 and 17). Sometimes, even if the probability of being in a healthy state is low (and accordingly the DGA and transformer health indices are negatively affected), the ongoing fault identified by the DGA module is a lowimpact fault, such as low temperature thermal fault or low intensity electrical fault.

The traditional health index value is more conservative than the proposed health index formulation. The DGA fac- 
tor of the traditional health index (Figure 15) is more conservative than the novel DGA health index metric (Figure 18), but the effect of the load factor of the traditional health index (Figure 12) is more penalising than the insulation paper health index (Figure 11) due to the linear model in Eq. (18) based on monthly peak loads. This results in an overall more conservative health index estimate, except for the first sample. In the first sample of the proposed health index framework, the DGA health index is not 1 and this is caused by the probabilistic diagnostics at 15/10/12 in Figure 16 $(\operatorname{Pr}($ Normal $)=0.97)$ and the confidence bounds in Figure 11.

So as to adopt maintenance-related decisions, the engineer can make use of the transformer-level health index metric, or alternatively, the proposed independent subsystem health indices can be used so as to examine the health state of different subsystems. The propagation of the uncertainty estimates from the solid insulation health index (Figure 11) into the transformer-level health index (Figure 21) results in a worst case transformer health index variation of 0.01 units. This is informative to examine the effect of temperature and load measurement errors, as well as uncertain degradation processes on the solid insulation and transformer-level health state. If the maximum likelihood value was lower and the confidence bounds were wider, the decision-making process would have been more intricate.

In contrast, the expert-based health index formulation in [27] generates deterministic health index values without uncertainty modelling and without including many of the different DGA and insulation paper factors integrated in this work. Accordingly, the proposed health index model assists the engineer in the transformer maintenance decisionmaking processes with more informative and uncertainty-aware subsystem and system-level health index metrics. Depending on the magnitude of the errors, the effect of uncertainty in decision-making can become critical.

Comparison with other health index methods in Table 1 is complex because the proposed approach is not a supervised learning problem. That is why a subsystem-based approach has been selected to make direct comparisons [27], and where possible, subsystem-specific health index methods have been directly compared, i.e. DGA health index classifier in Table 4.

\section{Discussion}

Overall, it has been shown that different subsystems health indices have been validated independently and the combination of these modules is based on expert-based weighting methods. Different datasets have been used for different transformer subsystems.

For the insulation paper health index hourly sampled variables for three years and nine months have been used including cooling water, ambient temperature and load. This model is based on IEEE standard equations [14] and Bayesian tracking methods (Particle Filtering).

For the DGA classification model, the IEC TC10 standard DGA benchmarking dataset has been used which is comprised of 167 DGA data samples. This dataset is widely used in the DGA community to validate classifiers, e.g. $[49,50]$. Additionally, Monte Carlo Cross Validation has been used to infer the results, which trains and tests models $N$ times with randomly shuffled datasets, and enables the generalization of the results. After validating the model with the results shown in Subsection 4.2 the testing set has been used as shown in Figure 13.

The limits and values for the rate-of-change of gases and absolute levels have been elicited from [41].

Note that the designed DGA classification method may be replaced or enhanced with other classification strategies, if appropriate. This is not a limiting factor to apply the proposed DGA health index and the overall transformer health index methodology.

Finally, the oil health index is deliberately based on a expert-based weighted average method because there are not enough available data samples to design a data-driven analytics model. Namely, oil quality related data variables are limited to 8 samples (see Figure 20a) and this is the limiting factor when generating the transformer-level health index which includes information about all the transformer subsystems. However, the modularity and flexibility of the designed health index framework permits the independent evaluation of solid insulation and DGA health indices.

The proposed health index has been limited to the data sources available in the case study (cf. Figure 3). However, there are other subsystems and datasets that can be included to generate a more complete picture of the health of the transformer, such as furans, $\mathrm{CO} / \mathrm{CO}_{2}$, winding resistance, tap turns ratio, capacitance (see also Figure 1). In this context, again the modularity and flexibility of the framework makes it possible to extend the approach with datasets where available.

The applicability of the proposed health index framework is not limited to monitor the health state of the overall transformer, but it can also be used to monitor the health of different subsystems. Additionally, the effect of multiple 
uncertainty sources considered across different transformer subsystems is propagated to the transformer health index level which better informs the final decision making process and makes it suitable for application in the context of systems surrounded with measurement and process uncertainties.

In this case the framework has been applied to power transformers, but many of the modelling concepts are generic and they can be transferred to monitor other complex systems. However, the specific models depend on the system under study and available datasets or physics-of-failure degradation models.

\section{Conclusions}

Transformers are key assets for the reliable and cost-effective operation of the power grid. However, transformer condition monitoring is an intricate task. Transformers are comprised of complex and interacting subsystems surrounded by different sources of uncertainty. PHM strategies are gaining a constantly increasing significance and attention as possible health monitoring solutions for transformers. However, with the emergence of dynamic power and energy applications such as dynamic load changes and de-energized periods, there are different sources of uncertainty that affect the overall transformer health. Accordingly it is necessary to upgrade existing transformer PHM strategies from a systems engineering perspective with uncertainty modelling mechanisms.

In this context, this paper has presented a novel soft computing based health index framework for transformer health monitoring under uncertainty. The framework integrates transformer subsystem health indices for solid insulation, dissolved gas analysis and oil quality, which are modeled respectively through experimental and Bayesian Particle Filtering models; ensemble and expert-based models; and weighted average health state estimations. The integration of transformer subsystems has been performed through expert knowledge.

It has been demonstrated that when considering the effect of measurement errors from monitoring sensors and the lack of precise knowledge of stochastic degradation processes, the final health index value is more informative and valuable. The obtained results have been compared with traditional health index values and it has been shown that the proposed framework is a more complete specification and robust to measurement errors.

Future work may focus on the use of data-driven statistical methods to combine the health indices of different subsystems.

\section{Acknowledgements}

This research was funded by Bruce Power, Babcock International, EDF Energy and Kinectrics through the Advanced Nuclear Research Centre at the University of Strathclyde. All the authors are grateful to the Editor and the anonymous reviewers for their insightful feedback.

\section{Bibliography}

\section{References}

[1] M. J. Heathcote, J \& P Transformer Book, Thirteenth Edition, Newnes, 2007 (2007). doi:https://doi.org/10.1016/B978-075068164-3/50008-9.

[2] E. Hajipour, M. Mohiti, N. Farzin, M. Vakilian, Optimal distribution transformer sizing in a harmonic involved load environment via dynamic programming technique, Energy 120 (2017) 92 - 105 (2017).

[3] J. Aizpurua, V. Catterson, B. Stewart, S. McArthur, B. Lambert, B. Ampofo, G. Pereira, J. Cross, Determining appropriate data analytics for transformer health monitoring, in: NPIC-HMIT, 2017, pp. 1-11 (2017).

[4] V. Duraisamy, N. Devarajan, D. Somasundareswari, A. A. M. Vasanth, S. Sivanandam, Neuro fuzzy schemes for fault detection in power transformer, Applied Soft Computing 7 (2) (2007) 534 - 539 (2007). doi:https://doi.org/10.1016/j.asoc.2006.10.001.

[5] S. wei Fei, X. bin Zhang, Fault diagnosis of power transformer based on support vector machine with genetic algorithm, Expert Systems with Applications 36 (8) (2009) 11352 - 11357 (2009). doi:https://doi.org/10.1016/j.eswa.2009.03.022.

[6] N. Yadaiah, N. Ravi, Internal fault detection techniques for power transformers, Applied Soft Computing 11 (8) (2011) 5259 - 5269 (2011). doi:https://doi.org/10.1016/j.asoc.2011.05.034.

[7] A. Azmi, J. Jasni, N. Azis, M. A. Kadir, Evolution of transformer health index in the form of mathematical equation, Renewable and Sustainable Energy Reviews 76 (2017) 687 - 700 (2017). doi:https://doi.org/10.1016/j.rser.2017.03.094.

[8] A. Peimankar, S. J. Weddell, T. Jalal, A. C. Lapthorn, Multi-objective ensemble forecasting with an application to power transformers, Applied Soft Computing 68 (2018) 233 - 248 (2018). doi:https://doi.org/10.1016/j.asoc.2018.03.042.

[9] S. Kabir, An overview of fault tree analysis and its application in model based dependability analysis, Expert Systems with Applications 77 (2017) 114-135 (2017). doi:https://doi.org/10.1016/j.eswa.2017.01.058. 
[10] IEEE, IEEE Guide for the Evaluation and Reconditioning of Liquid Immersed Power Transformer, IEEE Std. C57.140-2006 (2006) (2006).

[11] H. Wu, G. K.-H. Pang, K. L. Choy, H. Y. Lam, Dynamic resource allocation for parking lot electric vehicle recharging using heuristic fuzzy particle swarm optimization algorithm, Applied Soft Computing 71 (2018) 538 - 552 (2018). doi:https://doi.org/10.1016/j.asoc.2018.07.008.

[12] M. Faheem, V. Gungor, Energy efficient and qos-aware routing protocol for wireless sensor network-based smart grid applications in the context of industry 4.0, Applied Soft Computing 68 (2018) 910 - 922 (2018). doi:https://doi.org/10.1016/j.asoc.2017.07.045.

[13] S. Al-Dahidi, F. D. Maio, P. Baraldi, E. Zio, R. Seraoui, A framework for reconciliating data clusters from a fleet of nuclear power plants turbines for fault diagnosis, Applied Soft Computing 69 (2018) 213 - 231 (2018). doi:https://doi.org/10.1016/j.asoc.2018.04.044.

[14] IEEE PES, IEEE Guide for Loading Mineral-Oil-Immersed Transformers and Step-Voltage Regulators, IEEE Std. C57.91 (2011) (2011).

[15] A. Seier, P. D. H. Hines, J. Frolik, Data-driven thermal modeling of residential service transformers, IEEE Trans. Smart Grid 6 (2) (2015) 1019-1025 (March 2015). doi:10.1109/TSG.2015.2390624.

[16] M. Schlechtingen, I. F. Santos, Wind turbine condition monitoring based on scada data using normal behavior models. part 2: Application examples, Applied Soft Computing 14 (2014) 447 - 460 (2014). doi:https://doi.org/10.1016/j.asoc.2013.09.016.

[17] H. Ma, T. K. Saha, C. Ekanayake, D. Martin, Smart transformer for smart grid - intelligent framework and techniques for power transformer asset management, IEEE Trans. Smart Grid 6 (2) (2015) 1026-1034 (March 2015). doi:10.1109/TSG.2014.2384501.

[18] E. Tsoutsanis, N. Meskin, M. Benammar, K. Khorasani, A dynamic prognosis scheme for flexible operation of gas turbines, Appl Energy 164 (2016) 686 - 701 (2016). doi:https://doi.org/10.1016/j.apenergy.2015.11.104.

[19] D. Zhou, H. Zhang, S. Weng, A novel prognostic model of performance degradation trend for power machinery maintenance, Energy 78 (2014) 740 - 746 (2014).

[20] D. Zhou, F. Gao, E. Breaz, A. Ravey, A. Miraoui, Degradation prediction of PEM fuel cell using a moving window based hybrid prognostic approach, Energy 138 (2017) 1175 - 1186 (2017).

[21] Y. Lin, X. Li, Y. Hu, Deep diagnostics and prognostics: An integrated hierarchical learning framework in phm applications, Applied Soft Computing Journal 72 (2018) 555-564 (2018). doi:10.1016/j.asoc.2018.01.036.

[22] F. Ding, Z. Tian, F. Zhao, H. Xu, An integrated approach for wind turbine gearbox fatigue life prediction considering instantaneously varying load conditions, Renewable Energy 129 (2018) 260-270 (2018). doi:10.1016/j.renene.2018.05.074.

[23] Y. Lei, N. Li, L. Guo, N. Li, T. Yan, J. Lin, Machinery health prognostics: A systematic review from data acquisition to rul prediction, Mechanical Systems and Signal Processing 104 (2018) 799 - 834 (2018). doi:https://doi.org/10.1016/j.ymssp.2017.11.016.

[24] E. Chiodo, D. Lauria, F. Mottola, C. Pisani, Lifetime characterization via lognormal distribution of transformers in smart grids: Design optimization, Appl Energy 177 (2016) 127 - 135 (2016). doi:https://doi.org/10.1016/j.apenergy.2016.04.114.

[25] D. Zhou, Z. Wang, P. Jarman, C. Li, Data requisites for transformer statistical lifetime modelling - Part II: Combination of random and aging-related failures, IEEE Trans. Pow. Del. 29 (1) (2014) 154-160 (Feb 2014). doi:10.1109/TPWRD.2013.2270116.

[26] Q. Zhang, C. Hua, G. Xu, A mixture Weibull proportional hazard model for mechanical system failure prediction utilising lifetime and monitoring data, Mechanical Systems and Signal Processing 43 (1) (2014) 103 - 112 (2014). doi:https://doi.org/10.1016/j.ymssp.2013.10.013.

[27] A. Jahromi, R. Piercy, S. Cress, J. Service, W. Fan, An approach to power transformer asset management using health index, IEEE Electr. Insul. Mag. 25 (2) (2009) 20-34 (March 2009). doi:10.1109/MEI.2009.4802595.

[28] W. H. Tang, K. Spurgeon, Q. H. Wu, Z. J. Richardson, An evidential reasoning approach to transformer condition assessments, IEEE Trans. Pow. Del. 19 (4) (2004) 1696-1703 (Oct 2004). doi:10.1109/TPWRD.2003.822542.

[29] A. E. B. Abu-Elanien, M. M. A. Salama, M. Ibrahim, Calculation of a Health Index for Oil-Immersed Transformers Rated Under 69 kV Using Fuzzy Logic, IEEE Trans. Pow. Del. 27 (4) (2012) 2029-2036 (Oct 2012). doi:10.1109/TPWRD.2012.2205165.

[30] A. D. Ashkezari, H. Ma, T. K. Saha, C. Ekanayake, Application of fuzzy support vector machine for determining the health index of the insulation system of in-service power transformers, IEEE Trans. Dielectrics and Electrical Insul. 20 (3) (2013) $965-973$ (June 2013). doi:10.1109/TDEI.2013.6518966.

[31] S. Li, H. Ma, T. Saha, G. Wu, Bayesian information fusion for probabilistic health index of power transformer, IET Generation, Transmission \& Distribution (2017) (August 2017). doi:10.1049/iet-gtd.2017.0582.

[32] S. Tee, Q. Liu, Z. Wang, Insulation condition ranking of transformers through principal component analysis and analytic hierarchy process, IET Generation, Transmission \& Distribution 11 (1) (2017) 110-117 (2017). doi:10.1049/iet-gtd.2016.0589.

[33] K. Benhamed, A. Mooman, A. Younes, K. Shaban, A. El-Hag, Feature selection for effective health index diagnoses of power transformers, IEEE Trans. Pow. Del. PP (99) (2017) 1-1 (2017). doi:10.1109/TPWRD.2017.2762920.

[34] K. Ibrahim, R. M. Sharkawy, H. K. Temraz, M. M. A. Salama, Selection criteria for oil transformer measurements to calculate the health index, IEEE Trans. Dielectrics and Electrical Insulation 23 (6) (2016) 3397-3404 (Dec 2016). doi:10.1109/TDEI.2016.006058.

[35] S. Sankaraman, K. Goebel, Uncertainty in prognostics and systems health management, International Journal Prognostics and Health Management 6 (10) (2015) 14 (2015).

[36] J. Aghaei, A. Ahmadi, A. Rabiee, V. G. Agelidis, K. M. Muttaqi, H. Shayanfar, Uncertainty management in multiobjective hydro-thermal self-scheduling under emission considerations, Applied Soft Computing 37 (2015) 737 - 750 (2015). doi:https://doi.org/10.1016/j.asoc.2015.08.046

[37] J. I. Aizpurua, S. D. J. McArthur, B. G. Stewart, B. Lambert, J. G. Cross, V. M. Catterson, Adaptive power transformer lifetime predictions through machine learning and uncertainty modeling in nuclear power plants, IEEE Transactions on Industrial Electronics 66 (6) (2019) 4726-4737 (June 2019). doi:10.1109/TIE.2018.2860532.

[38] M. S. Arulampalam, S. Maskell, N. Gordon, T. Clapp, A tutorial on particle filters for online nonlinear/non-Gaussian Bayesian tracking, IEEE Trans. Signal Process. 50 (2) (2002) 174-188 (Feb 2002). doi:10.1109/78.978374.

[39] B. Saha, K. Goebel, Model adaptation for prognostics in a particle filtering framework, Int. J. of Prognostics and Health Management 2 (6) (2011) 1-10 (2011)

[40] E. Zio, G. Peloni, Particle filtering prognostic estimation of the remaining useful life of nonlinear components, Reliability Engineering \& System Safety 96 (3) (2011) 403 - 409 (2011). doi:https://doi.org/10.1016/j.ress.2010.08.009.

[41] IEEE PES, IEEE Guide for the Interpretation of Gases Generated in Oil-Immersed Transformers, IEEE Std. C57.104 (2008) (2008).

[42] J. Liu, E. Zio, A svr-based ensemble approach for drifting data streams with recurring patterns, Applied Soft Computing 47 (2016) 553 - 564 
(2016). doi:https://doi.org/10.1016/j.asoc.2016.06.030.

[43] X. Feng, Z. Xiao, B. Zhong, J. Qiu, Y. Dong, Dynamic ensemble classification for credit scoring using soft probability, Applied Soft Computing 65 (2018) 139 - 151 (2018). doi:https://doi.org/10.1016/j.asoc.2018.01.021.

[44] D. Fixsen, R. P. S. Mahler, The modified Dempster-Shafer approach to classification, IEEE Trans. Syst. Man, Cybern. A: Syst., Humans 27 (1) (1997) 96-104 (Jan 1997). doi:10.1109/3468.553228.

[45] Q.-S. Xu, Y.-Z. Liang, Monte Carlo cross validation, Chemometrics and Intelligent Laboratory Systems 56 (1) (2001) 1 - 11 (2001). doi:https://doi.org/10.1016/S0169-7439(00)00122-2.

[46] M. Duval, A. dePablo, Interpretation of gas-in-oil analysis using new IEC publication 60599 and IEC TC 10 databases, IEEE Electr. Ins. Mag. 17 (2) (2001) 31-41 (March 2001). doi:10.1109/57.917529.

[47] L. Wang, X. Zhao, J. Pei, G. Tang, Transformer fault diagnosis using continuous sparse autoencoder, SpringerPlus 5 (1) (2016) 448 (Apr 2016). doi:10.1186/s40064-016-2107-7.

[48] Ieee guide for acceptance and maintenance of insulating mineral oil in electrical equipment, IEEE Std C57.106-2015 (Revision of IEEE Std C57.106-2006) (2016) 1-38 (March 2016). doi:10.1109/IEEESTD.2016.7442048.

[49] A. Shintemirov, W. Tang, Q. H. Wu, Power transformer fault classification based on dissolved gas analysis by implementing bootstrap and genetic programming, IEEE Trans. Systems, Man, and Cybern. C 39 (1) (2009) 69-79 (Jan 2009). doi:10.1109/TSMCC.2008.2007253.

[50] L. Wang, X. Zhao, J. Pei, G. Tang, Transformer fault diagnosis using continuous sparse autoencoder, SpringerPlus 5 (1) (2016). doi:10.1186/s40064-016-2107-7. 